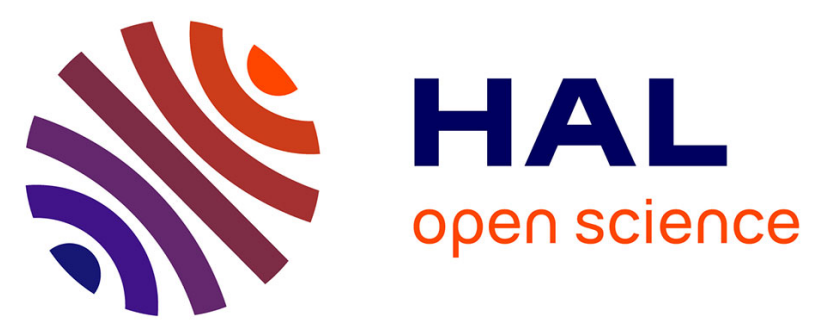

\title{
Simultaneous soot temperature and volume fraction measurements in axis-symmetric flames by a two-dimensional modulated absorption/emission technique
}

Guillaume Legros, Qianlong Wang, Jérôme Bonnety, Muhammad Kashif, Céline Morin, Jean-Louis Consalvi, Fengshan Liu

\section{To cite this version:}

Guillaume Legros, Qianlong Wang, Jérôme Bonnety, Muhammad Kashif, Céline Morin, et al.. Simultaneous soot temperature and volume fraction measurements in axis-symmetric flames by a twodimensional modulated absorption/emission technique. Combustion and Flame, 2015, 162, pp.2705 2719. 10.1016/j.combustflame.2015.04.006 . hal-01558554

\section{HAL Id: hal-01558554 https://hal.sorbonne-universite.fr/hal-01558554}

Submitted on 8 Jul 2017

HAL is a multi-disciplinary open access archive for the deposit and dissemination of scientific research documents, whether they are published or not. The documents may come from teaching and research institutions in France or abroad, or from public or private research centers.
L'archive ouverte pluridisciplinaire HAL, est destinée au dépôt et à la diffusion de documents scientifiques de niveau recherche, publiés ou non, émanant des établissements d'enseignement et de recherche français ou étrangers, des laboratoires publics ou privés. 


\title{
Simultaneous soot temperature and volume fraction measurements in axis-symmetric flames by a two-dimensional modulated absorption/emission technique
}

\author{
Guillaume Legros ${ }^{\mathrm{a}, \mathrm{b}, *}$, Qianlong Wang ${ }^{\mathrm{a}, \mathrm{b}}$, Jérôme Bonnety ${ }^{\mathrm{a}, \mathrm{b}}$, Muhammad \\ Kashifa,b,c, Céline Morin ${ }^{\mathrm{d}}$, Jean-Louis Consalvi ${ }^{\mathrm{e}}$, Fengshan Liu ${ }^{\mathrm{f}}$ \\ ${ }^{a}$ Sorbonne Univ, UPMC Univ Paris 06, UMR 7190, Inst Jean Le Rond dAlembert, \\ F-75005, Paris, France. \\ ${ }^{b}$ CNRS, UMR 7190, Inst Jean Le Rond dAlembert, F-75005, Paris, France. \\ ${ }^{c}$ University of Central Punjab, Department of Mechanical Engineering, Faculty of \\ Engineering, Johar Town, Lahore, Pakistan. \\ ${ }^{d}$ LAMIH CNRS UMR 8201, UVHC, F-59313 Valenciennes, France. \\ eAix-Marseille Université, IUSTI/ UMR CNRS 7343, F-13453 Marseille Cedex 13, \\ France. \\ ${ }^{f}$ Measurement Science and Standards, National Research Council of Canada, Ottawa, \\ Ontario, Canada.
}

\section{Abstract}

The original contribution of the present paper is to present a joint theoretical and experimental approach to implement the modulated absorption/emission technique. Two-dimensional fields of soot temperature and volume fraction can then be measured simultaneously in a reference steady laminar coflow axis-symmetric non-premixed ethylene flame estalished over

\footnotetext{
*Corresponding author

Email addresses: guillaume.legros@dalembert.upmc.fr (Guillaume Legros), wangqianlong212@gmail.com (Qianlong Wang), jerome.bonnety@upmc.fr (Jérôme Bonnety), muhammad.kashif@ucp.edu.pk (Muhammad Kashif), celine.morin@univ-valenciennes.fr (Céline Morin), jean-louis.consalvi@univ-amu.fr (Jean-Louis Consalvi), Fengshan.Liu@nrc-cnrc.gc.ca (Fengshan Liu)
} 
the Santoro burner. The spontaneous flame emission is captured by two CMOS cameras that allow the measurements of the flame emission intensity at two different wavelengths, i.e., $645 \mathrm{~nm}$ and $785 \mathrm{~nm}$ in this study. Concomitantly, two $70 \mathrm{~mm}$ diameter laser beams are used to pass through the flame, enabling the spectral line-of-sight attenuation to be imaged at the two wavelengths by the CMOS sensors. In these spectral ranges, both absorption and emission phenomena are dominated by soot. The local spectral absorption coefficient and spectral emission rate at both wavelengths are obtained by the onion-peeling method with Tikhonov regularization. The soot volume fraction map is inferred from the spectral absorption coefficient field. Mapping soot temperature does not require any model correlating soot volume fraction and local spectral absorption coefficient. Only the measurements of the latter at both wavelengths are required to enable the self-calibration of the technique and infer soot temperature from the ratio of the local spectral emission rates. Thus, the issue of the large discrepancies in the wavelength-dependent soot refractive index reported in the literature does not arise. Within the region of high soot temperatures, the results obtained by the methodology are in good agreement with numerical and experimental data available in the literature. The use of continuous wave lasers as the light sources enables future investigations in flickering flames where the phenomenon of intermittent soot release through the flame tip still needs to be better understood.

Keywords: Soot diagnostics, flame emissions, soot volume fraction, soot temperature 


\section{Introduction}

Formed along combustion processes, soot particles can be ultimately released into the atmosphere. The presence of these particles in urban air poses a serious public health problem even at low concentration [1]. Recent studies also highlight the critical role that soot particles may play in global warming due to their significant ability to absorb the incoming solar radiation [2]. Therefore, strategies aiming at the reduction of soot release by combustion devices are required.

Soot production is the result of two competitive processes, formation and oxidation. In the last four to five decades, models of both processes have been extensively developed. Some numerical models are capable of producing fair predictions of local soot concentrations [3-8]. However, the accuracy of these models seems to be strongly flame configuration dependent. While a highly comprehensive numerical approach by Blanquart and Pitsch [7] has been validated over a relatively wide range of fundamental laboratory flame configurations, the methodology requires a large database from each configuration studied.

The results obtained by Blanquart and Pitsch were essentially in good agreement with experimental measurements of soot volume fraction in several laminar flames. The joint Volume-Surface-Hydrogen model developed by the authors for soot formation was shown to predict the bell-shaped curve, i.e., the peak soot volume fraction in the flame first increases with increasing $T_{10}$ (the temperature at $10 \mathrm{~mm}$ above the burner) up to a threshold temperature and then decreases as $\mathrm{T}_{10}$ is further increased. Still, as highlighted by these authors, $\mathrm{T}_{10}$ remains an arbitrary key parameter of the simulations. 
In a numerical study conducted on ethylene diffusion flames, Liu et al. [8] extended their CFD code that incorporates both simplified soot chemistry and radiative transfer models. These authors needed to introduce two temperature-dependent correction factors in the soot oxidation schemes to successfully simulate the Smoke-Point for a given experimental configuration, i.e., the conditions that lead to the quenching of the soot oxidation process, therefore to soot release through the flame tip. These authors indicate that the exact physical and chemical processes associated with the modified soot oxidation rates are not fully understood and no guarantees are given that the model will be accurate in other configurations.

Recently, McEnally and Pfefferle [9] investigated potential methodologies to improve the Smoke-Point measurement. These authors assessed the relevance of the Yield Sooting Index (YSI) that quantifies the propensity of the fuel investigated to produce soot. YSI is inferred from direct measurements of peak soot volume fraction in a reference axis-symmetric diffusion flame. Kashif et al. [10] extended the consistency of the YSI methodology to blends of liquid fuels. While these authors reported some full soot volume fraction fields measured in the reference flame to extract the YSI [11], a recent numerical study by Consalvi et al. [12] showed that experimental soot temperature field is required to validate and improve the simulation, and to better understand the YSI methodology.

Thus, mapping soot temperature in these reference flames would allow further validation of sophisticated models of soot formation and oxidation. A considerable number of experimental studies have devoted efforts to the development of optical techniques to measure temperature in sooting flames. 
Indeed, such non-invasive diagnostics enable the reacting flows to be both probed without any perturbation and mapped potentially over a quite large area. Recent investigations [13-15] showed that the decay signal provided by Laser Induced Incandescence (LII) can give access to the temperature of laser heated soot particles. Nonetheless, the use of two-color or spectrally resolved LII to infer the soot temperature during or after laser heating requires a careful characterization of the wavelength dependence of the soot emissivity [15]. Furthermore, Goulay et al. [15] mentioned that some physico-chemical processes are likely to occur at the surface of the soot during the laser heating, leading to large uncertainties in the evaluation of the actual soot temperature.

As an alternative, the multicolor pyrometry imaging of flame spontaneous emission is also widely applied to both laboratory flames [16-20] and practical devices [21, 22]. Based on flame emissions detected by a spectrometer in steady laminar sooting axis-symmetric coflow diffusion flames, Liu et al. [19] extended the pyrometry methodology to multi-wavelength local measurements to reconstruct the soot temperature and volume fraction fields. On a similar flame configuration, Zhao et al. [20] developed the Cone Beam Tomographic Three Colour Spectrometry using a CMOS color camera. This technique allows the green, blue, and red intensities emitted by the soot particles to be discriminated. Maps of soot temperature, volume fraction and mean particle diameter can then be obtained. These techniques require sophisticated data processing as the raw information captured especially depend on both blackbody emission and emissivity, i.e., temperature and soot volume fraction, respectively. The influence of soot self-absorption on the soot volume fraction and temperature retrieval also requires a careful cor- 
rection for flames exhibiting a relatively high optical thickness. Moreover, the determination of soot temperature depends on the spectral dependence of the soot refractive index, a quantity that is a topic of ongoing debate as large discrepancies are reported in the literature [15, 19, 20].

To circumvent this issue, Jenkins and Hanson [23] developed the modulated absorption/emission (MAE) technique to probe a steady rich premixed ethylene/air flame established over a McKenna burner. In their experiments in this one-dimensional flame, a photomultiplier was used to detect both the flame spontaneous emission and the absorption of two laser beams through the flame at each laser operating wavelength, i.e., $830 \mathrm{~nm}$ and $1300 \mathrm{~nm}$. In this way, the local spectral soot absorption coefficients are directly evaluated at both wavelengths from the laser absorption measurements then used to infer the soot temperature from the ratio of the local spectral emission rates captured at these wavelengths. Using Mie theory in the Rayleigh limit, the spectral absorption coefficient can be converted to the local soot volume fraction with the help of a soot refractive index model. As highlighted by Jenkins and Hanson, the MAE technique provides self-calibrated temperature measurements, i.e., it is independent of the soot refractive index selected.

However, extension of the MAE technique to two-dimensional fields has not been reported in the literature. The main contribution of the present paper is the development of the MAE technique allowing the simultaneous measurements of soot temperature and volume fraction distribution in a steady laminar axis-symmetric non-premixed ethylene flame. To this end, the theoretical methodology that inherently accounts for the soot self-absorption is first described. The numerical distributions of soot temperature and volume 
fraction provided by Blacha et al. [24] are used to compute the theoretical flame emission signals captured by a matrix of pixels of a camera. Doing so, the relevance of the extended MAE technique and its limitations can be addressed through comparing the numerical fields with the retrieved ones. The experimental setup designed to implement the MAE technique is then outlined. By conducting MAE measurements in the flame established over the Santoro's coflow burner that was simulated by Blacha et al., we finally show that the experimental profiles of soot temperature and volume fraction obtained by the MAE technique agree fairly well with the data available in the literature. The self-calibration of soot temperature can also be used to infer soot temperature maps from the one-color fields of local spectral emission rate.

\section{Theoretical methodology}

\subsection{Geometrical configuration}

The methodology is designed for an axis-symmetric configuration. In the following, $(\mathrm{O} z)$ is the flame's axis of symmetry. Its origin is located at the burner tip, defining the height above the burner (HAB). $r$ is the distance from the axis of symmetry. Figure 1 displays a schematic of a flame's cross-section at a given $z$. For symmetry reason, the quantities, such as the temperature $T$ and the spectral absorption coefficient $\kappa$, depend on the cylindrical coordinates $r$ and $z$, but not on the angular position $\theta$. Defining the cartesian coordinates, the $x$-axis is set parallel to the sensor $\mathrm{S}_{1}$ and the $y$-axis is normal to $\mathrm{S}_{1}$. 


\subsection{Physical model}

We consider the flame as an emitting, absorbing, but non-scattering medium. For the laminar coflow ethylene/air diffusion flame studied, the flame radiative spectrum in the visible is governed by the continuum radiation from soot, which is particularly true in the upper part of the visible spectrum. Moreover, absorption by soot particles is shown to be at least one order of magnitude higher than scattering, especially at large wavelengths in the visible [14].

In such a configuration, the Radiative Transfer Equation (RTE) that models the transfer of the radiative intensity $L_{\lambda}(s, \mathbf{u})$ in a direction $\mathbf{u}$ at wavelength $\lambda$ along an optical pathway $s$ can be expressed as follows [25]:

$$
\frac{d L_{\lambda}(s, \mathbf{u})}{d s}=\kappa_{\lambda}(s)\left[B_{\lambda}(s)-L_{\lambda}(s, \mathbf{u})\right]
$$

where $\kappa_{\lambda}$ is the local spectral absorption coefficient and $B_{\lambda}$ the spectral blackbody radiative intensity at the local temperature at the location $s$. This intensity is given by the Planck's law:

$$
B_{\lambda}=\frac{2 h \pi c^{2}}{\lambda^{5}\left(e^{\frac{h c}{\lambda k T}}-1\right)}
$$

with $k=1.380710^{-23} \mathrm{~J} / \mathrm{K}$ being the Boltzmann constant, $h=6.62610^{-34} \mathrm{~J} . \mathrm{s}$ the Planck constant, and $c$ the speed of light in the medium, which can be considered as that in vacuum $\left(2.99810^{8} \mathrm{~m} / \mathrm{s}\right)$ in the present study.

Figure 1 shows a schematic of a straight optical pathway $(s=y)$ through the axis-symmetric flame. The discretized fields of temperature $T$ and spectral absorption coefficient $\kappa$ are shown. The discretization of the fields is conditioned by the signal collection on the row of pixels in $\mathrm{S}_{1}$. The optical 
setup on the right is schematically composed of a set of convergent lenses $\mathrm{L}_{1}$ and $\mathrm{L}_{2}$ separated by a pinhole $\mathrm{P}_{1}$. This optical arrangement allows the flux $\phi$ conveyed along the straight optical pathways parallel to the $y$-axis to be captured on a row of pixels located on the sensor $S_{1}$. As an illustration, the red ray of a collimated laser beam follows the line-of-sight parallel to the $y$-axis. This ray conveys an incoming flux $\phi_{3}^{(0)}$ that is both partially absorbed along its pathway and augmented by the radiative emission of the medium. The incident flux $\phi_{3}$ is then captured by the third pixel starting from the location $x=0$ of the flame axis projected on $\mathrm{S}_{1}$.

As shown in Eq.(1), the positive contribution to the radiation intensity along this pathway is due to the blackbody intensity. At a given wavelength $\lambda$, this contribution results in an overall flux $\phi_{\lambda}^{e m i}$ accumulated and potentially self-absorbed along the pathway. Integrating this positive contribution in Eq.(1) over the detection wavelength bandwidth $\Delta \lambda$ and along the line-ofsight leads to the following expression:

$\phi_{\lambda}^{\text {emi }}=\Delta \lambda \Delta x^{p i x} \Delta z^{p i x} \eta_{\lambda}^{\text {coll }} \int_{y_{\min }}^{y_{\max }} \kappa_{\lambda}(y)\left(\int_{\Omega(y)} B_{\lambda}(y) d \Omega\right) e^{-\int_{y}^{y_{\max }} \kappa_{\lambda}\left(y^{\prime}\right) d y^{\prime}} d y$

In arriving at Eq. (3) it is assumed that the detection wavelength bandwidth is sufficiently narrow (typically $20 \mathrm{~nm}$ ) so that the blackbody intensity remains nearly constant. In Eq.(3) $\Delta x^{p i x}$ and $\Delta z^{p i x}$ are the impinged pixel's lengths along the $x$ and $z$ axes, respectively. $y_{\min }$ and $y_{\max }$ are the boundaries along the $y$-axis between which the participating medium (flame) is encompassed. $\eta_{\lambda}^{\text {coll }}$ is the overall efficiency of the collection optics. The portion of $B_{\lambda}$ that impinges on the pixel considered is collected over the solid angle $\Omega$. 
While this angle theoretically depends on the distance to the pixel, therefore on $y, \Omega$ can be considered a constant $\Omega^{\text {coll }}$ when this distance is two orders of magnitude higher than the characteristic pixel's length $\Delta x^{p i x}$. Furthermore, this portion of $B_{\lambda}$ is partially self-absorbed along the remaining pathway starting from $y$ as expressed by the exponential term.

With the aforementioned assumptions, the energy $\mathscr{E}_{\lambda}$ emi accumulated on the pixel during a time $\Delta t$ due to a constant impinging flux $\phi_{\lambda}^{e m i}$ can be expressed as follows:

$\mathscr{E}_{\lambda}^{e m i}=\Delta \lambda \Delta t \Delta x^{p i x} \Delta z^{p i x} \eta_{\lambda}^{\text {coll }} \Omega^{\text {coll }} \int_{y_{\min }}^{y_{\max }} \kappa_{\lambda}(y) B_{\lambda}(y) e^{-\int_{y}^{y_{\max }} \kappa_{\lambda}\left(y^{\prime}\right) d y^{\prime}} d y$

When an incoming flux $\phi_{\lambda}^{\text {laser }}$, i.e., $\phi_{3}^{(0)}$ shown in Fig.1, is provided by a non-coherent collimated laser beam, the corresponding energy $\mathscr{E}_{\lambda}$ iaser is superimposed to $\mathscr{E}_{\lambda}^{e m i}$ on the pixel. $\mathscr{E}_{\lambda}$ laser can be similarly derived:

$$
\mathscr{E}_{\lambda}^{\text {laser }}=\Delta t \Delta x^{\text {pix }} \Delta z^{\text {pix }} \eta_{\lambda}^{\text {coll }} \phi_{\lambda}^{\text {laser }} e^{-\int_{y_{\min }}^{y_{\max }} \kappa_{\lambda}(y) d y}
$$

In the above expression, $\Delta \lambda$ and $\Omega^{\text {coll }}$ are inherently included in the definition of the incoming flux $\phi_{\lambda}^{\text {laser }}$.

Thus, when the laser is on, the incoming energy $\mathscr{E}_{\lambda}^{(o n)}$ on each pixel is:

$$
\mathscr{E}_{\lambda}^{(\text {on })}=\mathscr{E}_{\lambda}^{\text {laser }}+\mathscr{E}_{\lambda}^{\text {semi }}
$$

Conversely, when the laser is off, the energy $\mathscr{E}_{\lambda}^{(o f f)}$ deposited on the pixel is entirely due to $\mathscr{E}_{\lambda}^{e m i}$ :

$$
\mathscr{E}_{\lambda}^{(o f f)}=\mathscr{E}_{\lambda}^{e m i}
$$


Measuring consecutively $\mathscr{E}_{\lambda}^{(o n)}$ and $\mathscr{E}_{\lambda}^{(o f f)}$ allows the difference between both quantities to be only connected to the spectral absorption coefficient field:

$$
\mathscr{E}_{\lambda}^{(\text {on })}-\mathscr{E}_{\lambda}^{(\text {off })}=\Delta t \Delta x^{p i x} \Delta z^{p i x} \eta_{\lambda}^{\text {coll }} \phi_{\lambda}^{\text {laser }} e^{-\int_{y_{\min }}^{y_{\max }} \kappa_{\lambda}(y) d y}
$$

Once the absorption coefficient field is retrieved by conducting a set of laser attenuation (or absorption when scattering can be neglected) measure-

ments based on $\mathscr{E}_{\lambda}^{(o n)}$, the measurement of $\mathscr{E}_{\lambda}^{(o f f)}$ is then directly connected to the temperature field, as shown by Eq.(4), which allows the reconstruction of the local spectral emission rate $\kappa_{\lambda} B_{\lambda}$, and hence the temperature field.

The soot volume fraction field $f_{v}$ can also be inferred. The Mie theory allows $\kappa_{\lambda}$ to be transformed into $f_{v}$, assuming that soot particles are in the Rayleigh limit [26]:

$$
f_{v}=\frac{\lambda \kappa_{\lambda}}{6 \pi E(m)}
$$

where $E(m)$ is a function of the complex refractive index $m$ of soot.

It is important to emphasize that the retrieval of the temperature field does not depend on the selection of the soot refractive index, since the absorption coefficient field is determined through laser attenuation measurements. This statement highlights the inherent advantage of the MAE technique. However, to the authors' knowledge, this methodology has not been formulated for two-dimensional fields.

\subsection{Mathematical formulation}

The line-of-sight MAE technique needs to be combined with a subsequent deconvolution method to compute the local fields that contribute to the detected signals. While few sophitiscated strategies have been designed to 
reconstruct three-dimensional fields [27], numerous numerical methods have been developed for axis-symmetric configurations. An onion-peeling method is employed here. Indeed it naturally takes advantage of the grid that a digital camera provides. Furthermore, Daun et al. [28] showed that the onion peeling method combined with a Tikhonov regularization is less susceptible to measurement errors than the regular Abel three-point deconvolution. Figure 2 represents the meshing of half a flame cross-section that is conditioned by a row of $N$ pixels ( $N=6$ here). Every $I_{i j}$ is the rendering of the energy accumulated along the line-of-sight parallel to the $y$-axis and impinging on the $\mathrm{j}^{\text {th }}$ pixel of the $\mathrm{i}^{\text {th }}$ row shown here. This schematic is used to formulate the numerical problems allowing Eqs.(8) and (4) to be solved for $\kappa_{\lambda}$ and $T$, respectively. For the rest of Section 2.3, the subscript $\lambda$ indicating the wavelength dependence is omitted for clarity.

\subsubsection{Soot spectral absorption coefficient field}

The procedure to retrieve the soot spectral absorption coefficient $\kappa_{i j}$ at every meshpoint $\left(r_{j}, z_{i}\right)_{j=1, N / i=1, M}$ has been extensively documented in the literature, e.g., Kashif et al. [26]. Only the main steps are briefly recalled.

At any height $\left(z_{i}\right)_{i=1, M}$ above the burner imaged on the $\mathrm{i}^{\text {th }}$ line of the sensor (see Fig.2), Eq.(8) is discretized as follows at every meshpoint $x_{j}$ on the $x$-axis of the sensor:

$$
2 \sum_{k=1}^{N} A_{j k}^{O P} \kappa_{i k}=-\log \left(\frac{\mathscr{E}_{i j}^{(o n)}-\mathscr{E}_{i j}^{(o f f)}}{\mathscr{E}_{i j}^{(o n, 0)}-\mathscr{E}_{i j}^{(o f f, 0)}}\right)
$$

The Right Hand Side (RHS) term in Eq.(10) is provided by the measurements of the energy deposited on the pixel located on the $\mathrm{i}^{\text {th }}$ line and the 
$\mathrm{j}^{\text {th }}$ column. The superscript 0 indicates the measurements in the absence of the flame. Due to the ratio in the logarithm, the experimental constants in the RHS term of Eq.(8) cancel out. The summation on the left hand side of Eq.(10) is performed along the line-of-sight.

Expressed at every meshpoint $\left(x_{j}\right)_{j=1, N}$, Eq.(10) leads to a system of $N$ linear equations, $2 \mathbf{A}^{O P} X=b$, that can be solved for $X=\left(\kappa_{i k}\right)_{k=1, N}$, with $b$ being the vector composed of $N$ RHS terms in Eq.(10). Then, to compute the whole field of soot absorption coefficient $\left(\kappa_{i k}\right)_{i=1, M / k=1, N}, M$ sets of $N$ linear equations need to be solved.

At any height $z_{i}$ above the burner, $\mathbf{A}^{O P}$ is a matrix composed of chord lengths that can be readily calculated using simple geometrical considerations:

$A_{j k}^{O P}= \begin{cases}0 & \text { if } j>k \\ \Delta x^{p i x} *\left[k^{2}-(j-0.5)^{2}\right]^{0.5} & \text { if } j=k \\ \Delta x^{p i x} *\left(\left[k^{2}-(j-0.5)^{2}\right]^{0.5}-\left[(k-1)^{2}-(j-0.5)^{2}\right]^{0.5}\right) & \text { if } j<k\end{cases}$

Typically, with the optical arrangement specified in Section 4 later, $\mathbf{A}^{O P}$ is an $80 x 80$ upper triangular matrix. Therefore, $\left(\kappa_{i k}\right)_{k=1, N}$ might be solved using the simple back-substitution. However, the aforementioned set of equations can be shown to be ill-conditioned. As recommended by Akesson and Daun [29], a Tikhonov regularization can be used to stabilize the deconvolution process. Following their procedure, the value of the regularization parameter $\Lambda$ is selected so that it is located in the corner of the $L$-curve obtained when plotting $\log _{10}\left(\left\|\mathbf{A}^{O P} X_{\Lambda}-b\right\|_{2}\right)$ versus $\log _{10}\left(\left\|\mathbf{L} X_{\Lambda}\right\|_{2}\right) . X_{\Lambda}$ is 
the regularized solution and $\mathbf{L}$ is a discrete approximation of the $\nabla$-operator.

\subsubsection{Soot temperature field}

Following a similar procedure, a discrete formulation of Eq.(4) can be derived. At every height $z_{i}$ above the burner, a linear system $\mathbf{C} X=d$ is solved for $X=\left(\kappa_{i j} B_{i j}\right)_{j=1, N}$ once the soot spectral absorption coefficient field $\left(\kappa_{i j}\right)_{j=1, N}$ has been retrieved. This should then allow $B_{i j}$, and therefore $T_{i j}$ (see Eq.(2)), to be computed at every meshpoint $\left(r_{j}, z_{i}\right)_{j=1, N / i=1, M}$.

Due to soot self-absorption along the line-of-sight, the contributions of the left and right quarters to any signal $I_{i j}$ represented in Fig.2 are not identical. Therefore, some algebraic manipulations are needed to formulate a system of $N$ linear equations.

As an illustration, we consider that the signal $I_{i 4}$ is a linear function of the energy deposited on the pixel considered, introducing $\zeta$ as the proportionality factor:

$$
I_{i 4}=\zeta \mathscr{E}_{i 4}^{e m i}
$$

According to Eq.(4), this energy scales as the cumulated contributions of the emitting parts of the flame along the line-of-sight. Therefore, summation of contribution of each segment along the line-of-sight shown in Fig.2, starting 
from the right to the left, $I_{i 4}$ can be expressed as follows:

$$
\begin{aligned}
& I_{i 4} \propto A_{46}^{O P} \kappa_{i 6} B_{i 6}+A_{45}^{O P} \kappa_{i 5} B_{i 5} e^{-\sum_{m=6}^{6} A_{4 m}^{O P} \kappa_{i m}}+A_{44}^{O P} \kappa_{i 4} B_{i 4} e^{-\sum_{m=5}^{6} A_{4 m}^{O P} \kappa_{i m}} \\
&+ A_{44}^{O P} \kappa_{i 4} B_{i 4} e^{-\sum_{m=4}^{6} A_{4 m}^{O P} \kappa_{i m}}+A_{45}^{O P} \kappa_{i 5} B_{i 5} e^{-2 \sum_{m=4}^{4} A_{4 m}^{O P} \kappa_{i m}-\sum_{m=5}^{6} A_{4 m}^{O P} \kappa_{i m}} \\
&+A_{46}^{O P} \kappa_{i 6} B_{i 6} e^{-2 \sum_{m=4}^{5} A_{4 m}^{O P} \kappa_{i m}-\sum_{m=6}^{6} A_{4 m}^{O P} \kappa_{i m}}
\end{aligned}
$$

Rearranging the above equation written with $\left(\kappa_{i j} B_{i j}\right)_{j=1, N}$ as variables then leads to the following one:

$$
\begin{aligned}
I_{i 4} & \propto A_{44}^{O P} e^{-\sum_{m=5}^{6} A_{4 m}^{O P} \kappa_{i m}}\left(1+e^{-A_{44}^{O P} \kappa_{i 4}}\right) \kappa_{i 4} B_{i 4} \\
& +A_{45}^{O P} e^{-\sum_{m=6}^{6} A_{4 m}^{O P} \kappa_{i m}}\left(1+e^{-2 \sum_{m=4}^{4} A_{4 m}^{O P} \kappa_{i m}-A_{45}^{O P} \kappa_{i 5}}\right) \kappa_{i 5} B_{i 5} \\
& +A_{46}^{O P}\left(1+e^{-2 \sum_{m=4}^{5} A_{4 m}^{O P} \kappa_{i m}-A_{46}^{O P} \kappa_{i 6}}\right) \kappa_{i 6} B_{i 6}
\end{aligned}
$$

Thus, at every height $z_{i}$ above the burner, a general expression of the $\mathbf{C}$ 
elements can be derived as follows:

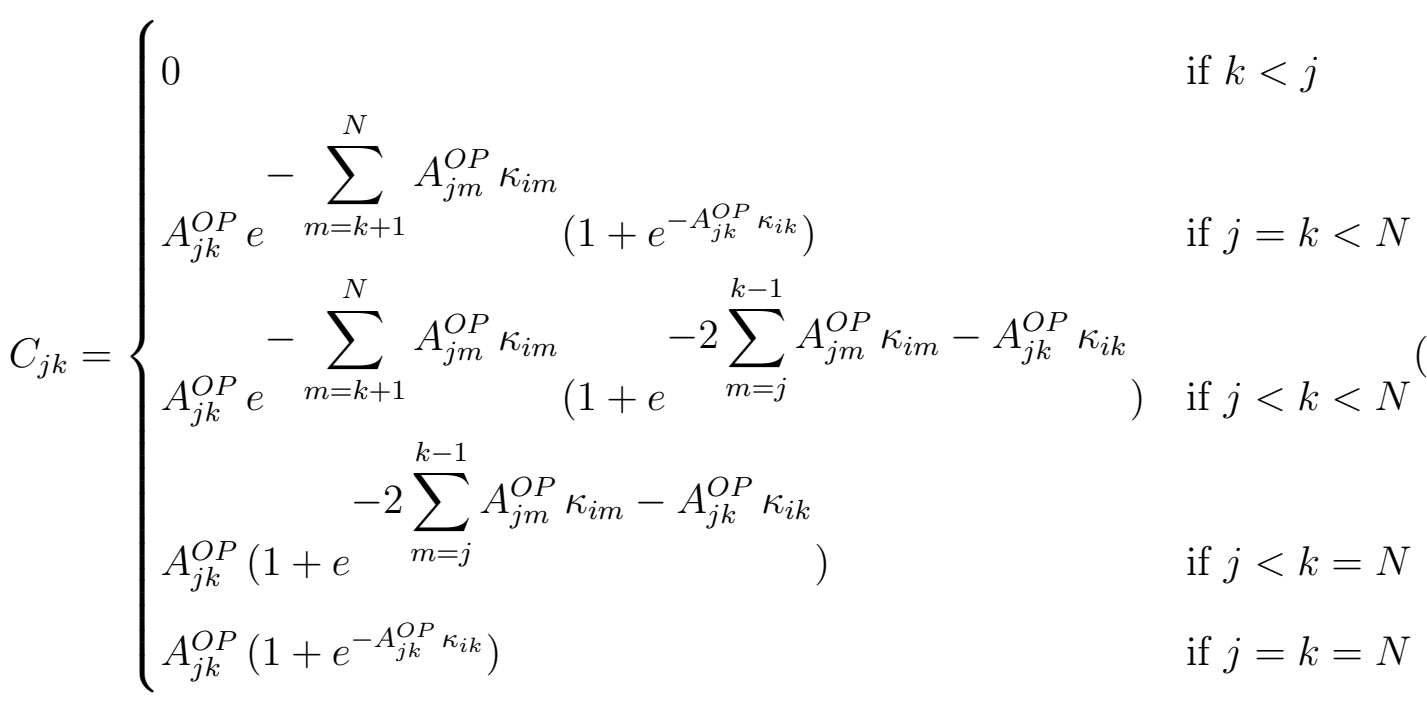

Then, at every height $z_{i}$, the system of $N$ linear equations appears as the following one:

$$
\mathrm{C} X=d
$$

where $\mathbf{C}$ is a lower triangular matrix and $X=\left(\kappa_{i j} B_{i j}\right)_{j=1, N}$. The vector $d$ can be expressed using Eqs.(4) and (12) as:

$$
d=\frac{1}{\zeta \Delta \lambda \Delta t \Delta x^{\text {pix }} \Delta z^{\text {pix }} \eta_{\lambda}^{\text {coll }}}\left(I_{i j}\right)_{j=1, N}
$$

By analogy with the procedure to retrieve the soot spectral absorption coefficient field, a Tikhonov regularization helps stabilize the deconvolution process represented by the system (16). The value of the regularization parameter is also selected using the aforementioned $L$-curve method.

The prior evaluation of the soot absorption coefficient field $\left(\kappa_{i j}\right)_{j=1, N}$ then allows the blackbody intensity field $\left(B_{i j}\right)_{j=1, N}$ to be inferred.

Because the detected flame emission signals expressed in Eq.(4) depend not only on the soot temperature and volume fraction distributions, but 
also on the detection system setup, it is difficult to reconstruct the soot temperature distribution from the detected emission signals. In practice, the flame emission signals at a second wavelength are required, since the ratio of the local emission terms at two different wavelengths will be uniquely related to the local temperature.

Finally, it is worth noticing that the interpretation of the soot spectral emission rate field expressed by Eq.(16) intrinsically includes soot selfabsorption. On the opposite, a methodology following the conventional twocolor pyrometry technique would require a specific processing to account for this phenomenon that can significantly affect the signals interpreted $[19,30,31]$.

\section{Methodology assessment}

As a benchmark, the fields of soot volume fraction $f_{v}$ and soot temperature $T$ numerically modeled by Blacha et al. [24] are used to assess both the consistency and the relevance of the methodology described in Section 2.

\subsection{Flame configuration}

Blacha et al. [24] modeled the flame experimentally investigated by McEnally et al. [37]. The steady axis-symmetric laminar diffusion flame is established over the Santoro's coflow burner at atmospheric pressure and room temperature $\left(20^{\circ} \mathrm{C} \pm 0.5^{\circ} \mathrm{C}\right)$. The central and annular flows are composed of ethylene and air, respectively. The ethylene and air flow rates are 3.85 $\mathrm{cm}^{3} / \mathrm{s}\left( \pm 0.02 \mathrm{~cm}^{3} / \mathrm{s}\right)$ and $770 \mathrm{~cm}^{3} / \mathrm{s}\left( \pm 1.4 \mathrm{~cm}^{3} / \mathrm{s}\right)$, respectively.

The paper by Blacha et al. [24] made a direct comparison of the numerical and experimental profiles of soot temperature and volume fraction. 
Therefore, the temperature and soot volume fraction distributions constitute a relevant database to evaluate the performance of the MAE technique in its two-dimensional formulation.

Furthermore, the flame selected exhibits strong radial variations in both temperature and soot volume fraction within the flame height of $50 \mathrm{~mm}$. As such, the main features of soot temperature and volume fraction distributions in this flame can be captured by the $70 \mathrm{~mm}$ diameter laser beam (see Section 4 below).

\subsection{Self-consistency of the methodology}

The first assessment consists in how well the technique can recover the assumed fields of soot temperature and volume fraction using numerically generated emission and transmission signals. To this end, 8 theoretical fields are numerically produced over a typical matrix of $M=1312$ lines and $N=1082$ columns of pixels, i.e., the simulated signals at both wavelengths, with and without laser illumination, and with and without the flame (see Eq.(10)).

For this specific assessment only, the signals $I_{i j}$ that would be collected as shown in Fig.2 are directly interpreted as the levels of energy deposited on the pixels. Therefore, their theoretical expressions are given by Eqs.(6) and (7). For both wavelengths, the signal without laser illumination and without

flame is set to zero. The parameters $\Omega^{\text {coll }}$ and $\phi_{\lambda}^{\text {laser }}$ are selected as such that lead to similar levels of peak $\mathscr{E}_{\lambda}^{\text {elaser }}$ and $\mathscr{E}_{\lambda}$ emi .

Soot volume fraction and temperature are provided at all meshpoints of the numerical domain simulated by Blacha et al. [24]. From these discrete fields, continuous functions $f_{v}(r, z)$ and $T(r, z)$ can be derived using a local cubic spline interpolation algorithm [33]. For every function, this algorithm 
minimizes the least-squares between the interpolating function and the whole set of corresponding numerical data. The continuous functions $f_{v}(r, z)$ and $T(r, z)$ are then found to adequately represent the numerical data with errors less than $3 \%$ and $0.5 \%$, respectively.

Using Eqs.(2) and (9), the continuous functions $\kappa_{\lambda}(r, z)$ and $\kappa_{\lambda} B_{\lambda}(r, z)$ can then be defined. At this point, the selection of both operating wavelengths and the associated values of $E(m)$ is required in order to generate the emission signals. In this study, the selected wavelengths are $\lambda_{1}=645 \mathrm{~nm}$ and $\lambda_{2}=785 \mathrm{~nm}$. At these wavelengths, the values of $E(m)$ are respectively 0.227 and 0.219 according to the soot refractive index of Chang and Charalampopoulos [34]. While the assessment of the methodology may depend on the wavelengths selected, it can be shown to be insensitive to the choice of the soot refractive index. In the remaining part of Section 3, both aforementioned continuous functions are considered as the fields to be retrieved by the MAE technique, expressed in Eqs.(8) and (4), by inverting the numerical signals based on these continuous functions.

Following the conceptual representation of the signal collection shown in Fig.2, the signals $I_{i j}$ can be computed using the analytical formulations expressed by Eqs.(8) and (4). To do so, at any wavelength and any pixel, Romberg integrations are performed, providing the numerical signals with and without laser. Then, these signals allow the RHS terms in Eqs.(10) and (16) to be computed. These systems are finally solved, providing the fields of spectral absorption coefficients $\left(\kappa_{i j}\right)_{i=1, M / j=1, N}$ and local spectral emission rates $\left(\kappa_{i j} B_{i j}\right)_{i=1, M / j=1, N}$.

Figure 3 shows the correlation between the retrieved spectral absorption 
coefficients (on the left) and local spectral emission rates (on the right) at wavelength $\lambda_{1}$ and the original numerical data (the inputs). All the dots (reconstructed values) are very closed to the blue lines (the input values). Very similar results are found at wavelength $\lambda_{2}$. Therefore, the MAE technique is shown to be self-consistent, as expected.

\subsection{Robustness of the methodology}

Different effects may affect the intrinsic performance of the MAE technique. Several factors can influence the conversion of the energy deposited on each pixel to the digital signals. These effects can be modeled as a sequence of three consecutive biasing processes [35]:

Step 1: The range of energy that can be detected is encompassed between a lower and an upper boundaries. The former represents the energy offset that needs to be deposited to generate a signal. The latter indicates the energy level above which saturation of the sensor occurs.

Step 2: Between these boundaries, the signal produced by the sensor can be decently modeled as a linear function of the energy deposited. However, the signal will be rounded by the analog to digital converter.

Step 3: Noise is generated along the aforementioned processes. Still it can be modeled as an additional random signal affecting the digital output at each pixel. 
Specifying the detection system characteristics allows these processes and their effects on the 8 fields of theoretical signals computed in Section 3.2 to be quantified. Based on the experimental design of this study described in Section 4, the following parameters related to the signal detection are considered appropriate:

Step 1: For any wavelength, the lower boundary is set at $10 \%$ of the peak $\mathscr{E}_{\lambda, i j}^{e m i}$. The upper boundary represents $140 \%$ of the peak $\left(\mathscr{E}_{\lambda, i j}^{\text {Ilaser }}+\mathscr{E}_{\lambda, i j}^{\mathscr{e} e m i}\right)$. Between both boundaries, the signal is equal to the energy deposited on the pixel.

Step 2: This range of energy is converted into a discrete scale spanning between 0 and 3000 (arbitrary unit).

Step 3: The mean background noise is set to 250 a.u. all over any $M \mathrm{x} N$ matrix of pixels. For each of the aforementioned 8 fields of theoretical signals, a matrix of gaussian noise with a zero mean and a standard deviation of 50 a.u. is added. This level of gaussian noise represents an upper boundary of the noise that could be experimentally characterized.

Once the 8 theoretical fields have been perturbed, $\left(\kappa_{i j}\right)_{i=1, M / j=1, N}$ and $\left(\kappa_{i j} B_{i j}\right)_{i=1, M / j=1, N}$ can be computed at both wavelengths $\lambda_{1}$ and $\lambda_{2}$. Then the ratio $\alpha$ of the blackbody intensities can be inferred from:

$$
\alpha_{i j}=\left(\frac{\kappa_{i j} B_{i j}}{\kappa_{i j}}\right)_{\lambda_{1}}\left(\frac{\kappa_{i j}}{\kappa_{i j} B_{i j}}\right)_{\lambda_{2}}
$$

The subscript of the brackets indicates here the wavelength at which the fields have been evaluated. The theoretical expression of the blackbody inten- 
sity formulated at both wavelengths (see Eq.(2)) allows the local temperature $T_{i j}$ to be inferred from $\alpha_{i j}$.

Figure 4 displays the correlation between the initial numerical input and the retrieved values for soot volume fraction (on the left) and temperature (on the right). The former field is obtained from the local spectral absorption coefficient retrieved at wavelength $\lambda_{1}$ using Eq.(9) and the soot refractive index of Chang and Charalampopoulos [34]. Similar results are found at wavelength $\lambda_{2}$. The latter field is inferred from the field of ratio $\alpha$. For the soot volume fraction, the retrieved data scatter quite uniformly along the ideal blue line. When monitoring the scattering along Step 1 to Step 3, the noise addition (Step 3) could be identified as the main contribution to the error in the soot volume fraction. Indeed, the noise characteristics do not depend on the location within the flame. On the other hand, the retrieved temperatures exhibit reduced levels of scattering with increasing temperature. The analog to digital conversion (Step 2) is here the biasing process that mainly contributes to the error in the local spectral emission rate. The rounding approximation is constant over the range of $\left(\kappa_{i j} B_{i j}\right)_{i=1, M / j=1, N}$ investigated, therefore, leading to higher relative errors at lower values of the local spectral emission rate.

Figure 5 shows the distributions of numerical data (on the left), results retrieved by the MAE technique when the signals have been perturbed (in the middle), and a selection of contours of the absolute discrepancy between the numerical and retrieved data (on the right). The upper and lower rows show the results for soot volume fraction and soot temperature, respectively. Only the temperature at the locations where the soot volume fraction is 
higher than $10 \%$ of the peak soot volume fraction is mapped. To obtain the soot volume fraction from the spectral absorption coefficient, the soot refractive indices of Chang and Charalampopoulos [34] have also been used. The field of soot volume fraction shown in Fig. 5 is inferred from the absorption coefficient of the red band $(645 \mathrm{~nm})$. As already mentioned, similar results are obtained with the absorption coefficient of the infrared band (785 $\mathrm{nm})$. As compared to the absolute levels of soot volume fraction and soot temperature, quite low levels of error are achieved by the MAE technique within the flame region where both the spectral absorption coefficients and the local spectral emission rates are relatively high. As an illustration, the contours delineating the regions where the soot volume fraction and the soot temperature discrepancies are lower than $0.5 \mathrm{ppm}$ and $40 \mathrm{~K}$, respectively, are relatively wide. This sensitivity analysis to the level of experimental noise shows that the MAE technique is significantly robust.

\subsection{Uncertainties evaluation and confidence regions}

The knowledge of the original fields especially allows the identification of regions where the methodology is able to deliver measurements with acceptable uncertainties. This section aims to determine the ranges of retrieved data that characterize these confidence regions.

The evaluation of the uncertainties related to soot volume fraction has already been documented in Ref.26. A major source of error can be attributed to the uncertainty in the soot refractive indices reported in the literature. In addition, the process to retrieve the soot spectral absorption coefficient field also contributes to the overall uncertainty. As the original contribution of the present paper consists in retrieving soot temperature, the evaluation of 
the uncertainties related to this quantity is now outlined. By analogy, the uncertainties in the spectral absorption coefficients can also be derived.

As shown by the maps of the retrieved data in Fig.5, the deconvolution procedure, even regularized, still leads to significant noise propagation to the flame's axis. Moreover, as a result of the perturbed red and infrared signals by the uncorrelated noise, the ratio of the two blackbody intensities exhibits locally uneven distribution within the region where both the local spectral emission rates are relatively high. This statement is illustrated in Fig.5 by the contours of the absolute discrepancy between the original temperature of Blacha et al. [24] and the retrieved one. While the contour defined by a discrepancy of $40 \mathrm{~K}$ delineates a relatively wide region of the flame, the boundary encompassing the region exhibiting a discrepancy of $60 \mathrm{~K}$ is more uneven. In the following, the locations encompassed by the contour of a 40 $\mathrm{K}$ discrepancy is called the ratio confidence region ( $\mathrm{RCR})$.

The self-calibrated measurements of soot temperature as inferred by the ratio of the blackbody intensities can indeed be used as a calibration database for every local spectral emission rate provided that the locations of these selfcalibrated measurements are included in the aforementioned RCR. To do so, at every location $(i, j)$ inside RCR, a local correction factor $\beta_{i j}$ can be defined as follows:

$$
(\kappa B)_{i j}=\beta_{i j} \kappa_{i j} B_{i j}
$$

using the local spectral emission rate $(\kappa B)_{i j}$ and the local spectral absorption coefficient $\kappa_{i j}$ measured at the location $(i, j)$, together with the self-calibrated temperature to compute the local blackbody intensity $B_{i j}$. The essence of the correction factor is to ensure that the product $\kappa_{i j} B_{i j}$ can accurately represent 
the local emission rate $(\kappa B)_{i j}$. Then the distribution of $\beta_{i j}$ within RCR allows the mean value $\bar{\beta}$ to be computed, which mitigates the noise influence. For each local spectral emission rate, $\bar{\beta}$ can be used as the correction factor. This procedure has been applied in the calculation of two other fields of temperature as inferred from the red and infrared local emission rates.

Figure 6 displays the evolutions of the mean absolute discrepancy between the original and retrieved temperatures. The field of temperature is retrieved using either the ratio of the blackbody intensities or one of the local spectral emission rates. The original data along the horizontal axis in Fig.6 are then either the aforementioned ratio or the red or infrared local emission rate. To scale these distributions, the horizontal axis is normalized by the respective peak quantity and the vertical axis by the respective peak mean absolute discrepancy.

The evolution of the ratio of the blackbody intensities first decreases, but then exhibits a peak located around 0.9. This range of ratio leading to higher discrepancy is especially due to regions where the red emission rate is relatively high and the red absorption coefficient vanishes, or conversely, the infrared emission rate is relatively low and the infrared absorption coefficient is non-negligible (see Eq.(18)). Therefore, the RCR has to be identified by not only a range of the ratio itself, but also a range for every local spectral absorption coefficient. Inspecting the data inside RCR leads to the following 
conditions:

$$
\begin{aligned}
\frac{\kappa_{\lambda_{1}}}{\max \left(\kappa_{\lambda_{1}}\right)} & \geq 0.14 \\
\frac{\kappa_{\lambda_{2}}}{\max \left(\kappa_{\lambda_{2}}\right)} & \geq 0.17 \\
\frac{\alpha}{\max (\alpha)} & \geq 0.2
\end{aligned}
$$

To assess the relevance of this identification, Figure 7 shows the distribution of the mean absolute discrepancy between the original and retrieved temperatures. The latter have been inferred from the ratio of the blackbody intensities. The distributions are evaluated either in RCR (red triangles) or in the whole field (blue circles) provided that the data exhibit values that also stand within the ranges Eqs.(20-22). As evidenced by the agreement between both distributions, these prove to contribute to the same extent to the mean absolute discrepancy up to a level of $40 \mathrm{~K}$. Above this level, a discrepancy between both distributions appears. However, the population extracted from the whole field is then very low (lower than $2 \%$ ). Therefore, the conditions in Eqs.(20-22) can be considered relevant constraints allowing RCR to be identified in the whole field.

In contrast, the evolutions shown in Fig.6 for both local spectral emission rates decrease monotonically, following first a dramatic reduction. Therefore, each local spectral emission rate can be assigned with only one threshold above which the temperature is inferred with an uncertainty level lower than $\pm 40 \mathrm{~K}$. These thresholds are indicated by the circles in Fig.6:

$$
\begin{aligned}
\frac{\kappa_{\lambda_{1}} B_{\lambda_{1}}}{\max \left(\kappa_{\lambda_{1}} B_{\lambda_{1}}\right)} & \geq 0.013 \\
\frac{\kappa_{\lambda_{2}} B_{\lambda_{2}}}{\max \left(\kappa_{\lambda_{2}} B_{\lambda_{2}}\right)} & \geq 0.022
\end{aligned}
$$


Figure 8 shows the maps of soot temperature as inferred from the red (on the left) and the infrared (on the right) local emission rates. In Fig.8, the spatial extension of each map is such that it includes only measurements of the local spectral emission rate that exceed the thresholds given by the relationships expressed in Eqs.(23-24). The ratio confidence region is delineated by the black dotted line. As evidenced in Fig.8, the calibration of the one-color fields leads to a spatial extension of the confidence region for temperature measurements as these fields cover larger regions of soot temperature at a similar level of uncertainties (here $40 \mathrm{~K}$ ).

\section{Experimental implementation}

\subsection{Burner configuration}

The steady laminar diffusion flame is established over the Santoro's axissymmetric coflow burner. Ethylene is injected via a Bronkhorst EL-FLOW mass flow controller through the vertical central brass duct, which has an $11 \mathrm{~mm}$ effective diameter of injection. The coflowing oxidizer consists of air, whose mass flow rate is controlled by another Bronkhorst EL-FLOW device. The air flow is then introduced into the concentric $102 \mathrm{~mm}$ inner diameter brass cylinder. Further details about the burner can be found in [36].

\subsection{Optical diagnostics}

Figure 9 displays the schematic of the optical arrangement that has been specifically designed to implement the two-dimensional modulated absorption/emission technique. This design can be considered as an extension of the Laser Extinction Method (LEM) setup by Kashif et al. [26]. 
Two $100 \mathrm{~mW}( \pm 0.5 \mathrm{~mW})$ Spectra-Physics Excelsior CW Lasers operating at $645 \mathrm{~nm}(-5 /+7 \mathrm{~nm})$ and $785 \mathrm{~nm}(-5 /+5 \mathrm{~nm})$ were used as the continuous light sources. The selection of these wavelengths is driven by trade-off. On one hand, absorption and emission by soot particles must be detected at both wavelengths at a similar order of magnitude to enable their measurements over a decent range of the sensor's dynamics. As a result, imaging both absorption and emission on a conventional camera sensor requires the use of the upper part of the visible spectrum. This strategy also consolidates the predominance of absorption over scattering by soot particles, therefore the relevance of the physical model considered (see Section 2.2). On the other hand, the gap between both wavelengths needs to be large enough to increase the slope of the function linking the ratio of blackbody intensities at both wavelengths to the soot temperature. Fulfilling this requirement leads to an enhanced sensitivity of the temperature measurement. Although the selection of the operating spectral ranges can be further optimized, the aforementioned wavelengths $\lambda_{1}=645 \mathrm{~nm}$ and $\lambda_{2}=785 \mathrm{~nm}$ allow the original methodology to reconstruct simultaneously the distributions of soot temperature and volume fraction with acceptable uncertainties. The choice of these two wavelengths is also based on the consideration that CW Lasers operating at these specific wavelengths are commercially available.

Each $1 \mathrm{~mm}$ diameter beam passes through a half-wave plate and a Newport Oriel Electronic shutter that chops the source at a frequency tuned here at $25 \mathrm{~Hz}$. A polarizing cube beamsplitter is used to transmit the red laser beam and reflect the infrared one. This arrangement allows the merged outcoming beam to be a combination of both wavelengths. Furthermore, 
the intensity of each beam can be tuned independently adjusting the angle between each half-wave plate and the polarizing cube.

The single beam then enters an optical fiber that is shaken by a vibrational element. This strategy eliminates the influence of the incoming polarizations and avoid the coherent effects of speckle and diffraction.

Using the natural divergence at the fiber outlet, the subsequent set of lenses and mirrors constitutes a beam expander. This provides with a collimated beam with an outgoing diameter of $70 \mathrm{~mm}$. Inserted along the optical pathway, a polished neutral density filter further reduces the beam intensity while enhancing its spatial uniformity.

After passing through the flame, the beam is decollimated. Upstream the focal point a beam splitter allows the reflection of $50 \%$ intensity and the transmission of the other $50 \%$ at both wavelengths. For each laser beam, a pinhole with an aperture diameter of $800 \mu \mathrm{m} \pm 5 \mu \mathrm{m}$ is located at the focal point. This provides a telecentric configuration possessing depth invariant magnification and filters the slight beam steering due to the temperature gradient that could bias the deconvolution process. Every resulting virtual image formed is re-imaged by a Photon Focus MV1 12-bit progressive scan monochrome camera mounted with a conventional lens and equipped with a narrow band filter. To enable the monochromatic imaging, these filters are centered at $645 \mathrm{~nm}( \pm 2 \mathrm{~nm})$ and $785 \mathrm{~nm}( \pm 3 \mathrm{~nm})$, and exhibit a band width at one half the transmissivity maximum of $20 \mathrm{~nm}$ and $10 \mathrm{~nm}$, respectively.

Every CMOS camera array is composed of $1312 \times 1082$ pixels, providing a spatial resolution of $150 \mu \mathrm{m}( \pm 10 \mu \mathrm{m})$ for the projected data.

A digital pulse generator (DG) controls the occurrence and the duration 
of the CMOS exposure, together with the shutters opening. A frame grabber records the frames captured by the cameras at a rate of 25 frames per second. In the present study, the exposure time of both cameras is set to $\Delta t=30$ ms.

The inserts in Fig. 9 show typical frames imaging the red (on the left) and infrared (on the right) signals when the shutters are open and the flame is established.

\subsection{Experimental procedure}

Before being mounted as shown in Fig.9, both camera sensors have first been corrected for the slight difference in sensitivity among the pixels. To do so, a uniformly illuminated screen was imaged on each sensor. Each frame captured allows a 1312x1082 matrix of sensitivity correction factors to be evaluated.

Then the whole optical diagnostics has been arranged and adjusted as depicted in Fig.9. Some discrepancies of the optical characteristics between the red and infrared pathways can be expected. As an illustration, the transmissivity and the reflectivity of the beam splitter are provided by the manufacturer (Newport) with an uncertainty up to $\pm 5 \%$. For this reason, the aforementioned screen was also used to measure the actual range of the overall transmissivity along every pathway. To this end, a spectrometer is used to collect the light emitted by the screen. This was placed at the location of the burner. After the light has been transmitted to the location of each camera, the measured spectrum allows the band of the overall transmissivity to be specified. 
A Halogen calibration light source (OCEAN OPTICS,HL-3 plus-INTCAL) was then used. The beam delivered by this lamp can be imaged on a sensor to calibrate the intensity measured by the sensor in arbitrary unit against the absolute power per unit area and per unit wavelength. Figure 10 depicts the evolution of the power of the beam along the visible spectrum, together with the bands $\Delta \lambda_{1}$ and $\Delta \lambda_{2}$ of the overall transmissivity along the red and infrared pathways. Thus, the absolute power that is expected to impinge on a sensor within any spectral range can be computed. Within the framework of the present study, the lamp was inserted at the location of the flame. The beam was imaged by both CMOS sensors, providing an accurate measurement of the ratio of the parameters that are required for the evaluation of the RHS term in Eq.(16). These measurements were conducted for a set of nine exposure times $\Delta t$ ranging from $10 \mathrm{~ms}$ to $50 \mathrm{~ms}$. This leads to the following mean evaluation of the aforementioned ratio, called $\chi$ :

$$
\chi=\frac{\left(\zeta \Delta \lambda \Delta t \Delta x^{p i x} \Delta z^{p i x} \eta^{\text {coll }}\right)_{\text {red }}}{\left(\zeta \Delta \lambda \Delta t \Delta x^{p i x} \Delta z^{\text {pix }} \eta^{\text {coll }}\right)_{\text {infrared }}}=1.70 \pm 0.03
$$

Based on the specifications of the manufacturers, the evaluation of this ratio leads to a theoretical value of 1.46. The difference between these two values supports the need for an experimental evaluation of the two CMOS sensors.

Figure 11 shows the evolution with temperature of the ratio of the blackbody intensities as captured over the red and infrared spectral ranges by the CMOS sensors. The ratio has been corrected for the measured discrepancy $\chi$ between the red and the infrared pathways to the CMOS sensors. The solid line represents the database that is to be used as the look-up table. This allows the ratio measured at any location to be converted into temperature. 
The dashed lines indicate the evolutions of the ratio when $\chi$ deviates by \pm 0.03 , i.e., the standard deviation reported in Eq.(25). This leads to an uncertainty of $\pm 10 \mathrm{~K}$ in the temperature inferred by the look-up table process within the range of temperature investigated [1000 K, $2200 \mathrm{~K}]$. As the lookup process follows the deconvolution procedure, the contributions of both processes to the overall uncertainty are cumulative. The overall uncertainty is then $\pm 50 \mathrm{~K}$.

The experimental flame conditions are set to those reported in Section 3.1 to reproduce as close as possible those used in the numerical simulation to generate the fields used to assess the methodology performance. A $10 \mathrm{~s}$ long sequence of frames was recorded every minute during 10 minutes. For every sequence, the 8 mean frames required to process the data were computed. The flame axis was readily detected on the frame imaging the flame without a laser beam. The parts of the frames located on the right hand side of the axis were processed to solve the systems (10) and (16) for $\kappa_{\lambda}$ then $B_{\lambda}$ at both wavelengths. The results shown in the following were found to be hardly affected by the sequence or the flame's side selected.

\section{Results and discussion}

\subsection{Consistency of the experimental design}

Figure 12 displays the images of experimental intensity as captured by both cameras when the flame is on and the laser is off, together with the signals computed as specified in section 3.2 from the numerical results of Blacha et al. [24]. These authors reported some discrepancies between their numerical soot volume fraction and temperature fields and the measurements in the 
experimental study of McEnally et al. [37]. Reproducing the experimental conditions of Santoro et al. [38], Arana et al. [39] also reported discrepancies among experimental measurements of soot volume fraction that can be especially attributed to the flow features in the vicinity of the burner tip. As a result, the quantitative agreement between the experimental and numerical maps shown in Fig.12 is not expected. For this reason, the location $\left(r_{\max }, z_{\max }\right)$ of the maximum signal $I_{\max }$ in each field has been used to produce the non-dimensional axes $r / r_{\max }$ and $z / z_{\max }$. While discrepancies among $\left(r_{\max }, z_{\max }\right)$ appear, the topology of the scaled maps $I / I_{\max }$ exhibits strong similarities. Thus, the experimental design is able to capture to a large extent the processes leading to the signals generated by the flame. Consequently, the methodology developed within the framework of the present study to retrieve the fields of soot temperature and spectral absorption coefficient is believed to be adequate.

\subsection{Soot volume fraction fields}

Figure 13 shows the profiles of soot volume fraction at the three different heights above the burner where experimental data by Arana et al. [39] are provided. These authors also implemented a Laser Extinction Method. The shaded areas indicate the regions where the uncertainty on the soot spectral absorption coefficient is higher than $\pm 2.5 \mathrm{~m}^{-1}( \pm 0.5 \mathrm{ppm})$. This range represents about $10 \%$ of the peak absorption coefficient measured in the infrared spectral range. The confidence region related to the infrared absorption coefficient is indeed the more restricted one. The spectral absorption coefficients retrieved can then be converted into soot volume fractions using the expression of the soot refractive index of Chang and Charalampopoulos [34] as a 
function of wavelength. The error bar reported in Fig.13 in terms of soot volume fraction is here attributed only to the data processing outlined in Section 2.3.1, therefore, not to the uncertainty in the soot refractive indices reported in the literature.

At any height above the burner, the profiles provided by the MAE technique at both wavelengths prove to be very similar. While discrepancies with the numerical profiles by Blacha et al. [24] appear, the peak soot volume fractions measured in the present study agree well with the ones measured by Arana et al. [39], except at the highest HAB considered. At this height, the peak measured in the present study is shifted outward as compared to the one reported by Arana et al. [39]. Yet, the profiles match one another in the vicinity of the flame's axis.

Thus, while the evolution of the soot volume fraction profiles obtained by the MAE technique qualitatively agree with those reported in the literature, quantitative discrepancies exist. Arana et al. [39] faced a similar issue and had to fit the soot refractive index to closely reproduce the profiles of Santoro et al. [38]. As argued by Arana et al., the discrepancies among experimental data can be partly attributed to the high sensitivity of the onset of soot formation to the flow features in the vicinity of the burner tip. Another significant source of discrepancy is the evolution of soot refractive index with soot formation history [19]. The latter further highlights the advantage of the MAE technique since it provides soot temperature measurements that depend directly on soot spectral absorption coefficient inferred from laser absorption measurements. As such, it does not require any models for the wavelength dependence of the soot refractive index. 
As an illustration, Figure 14 compares the soot spectral absorption coefficient distributions. The color scale is intentionally coarse to enable the comparison of the contours from one map to another. The fields of absorption coefficient measured within the red and infrared spectral ranges are shown on the left and in the middle, respectively. The expression of the soot refractive index as a function of wavelength of Chang and Charalampopoulos [34] has been used to provide the modeled field of infrared absorption coefficient shown on the right. This expression has been re-calibrated here with the peak red absorption coefficient measured. While the experimental and modeled fields of infrared absorption coefficient exhibit strong similarities, discrepancies exist at the middle of the field. For instance, the region showing infrared absorption coefficients higher than $50 \mathrm{~m}^{-1}$ is significantly smaller in the experimental field. Conducting a conventional two-color pyrometry requires the use of such a model which would in turn lead to larger uncertainties in the temperature measurements.

\subsection{Soot temperature fields}

Figure 15 exhibits the profiles of soot temperature at the three different heights above the burner shown in Fig.13. While discrepancies in the soot volume fraction profiles are revealed in Fig.13, the self-calibrated temperature measurements that are located within the confidence region of the MAE technique appear to match the profiles delivered by the thermocouple used by

McEnally et al. [32]. Indeed, these authors conducted a thorough analysis of their thermocouple output, especially correcting it for the effect of radiative transfer. As the confidence region of the present temperature measurement inferred from the ratio of the blackbody intensities is conditioned not only 
by the range of ratio but also by the ranges of absorption coefficients at both wavelengths, the aforementioned match is expected in spite of the discrepancies in soot volume fractions. This further supports the relevance of the MAE technique.

As mentioned earlier, a few measurements close to RCR can still depart from the smooth evolution of the profiles, as illustrated by the temperature overshoot around $r=1 \mathrm{~mm}$ at $\mathrm{HAB}=40 \mathrm{~mm}$ in Fig.15. For this reason, the temperature field encompassed within RCR is used as a calibration database for the fields of local spectral emission rate at both wavelengths. The correction factors are then inferred for the red and infrared spectral ranges at every location inside RCR. The red and infrared correction factors averaged over RCR are 54.5 and 90.2, respectively. The consistency of these mean factors is supported by the low relative standard deviations among the distributions of these factors, i.e., 4.1 and 8.9, respectively.

Finally, Figure 16 shows the maps of soot temperature as inferred from the red and the infrared local emission rates. The spatial extension of each map is such that it only includes measurements that exceed the thresholds of local emission rate determined in the theoretical approach (see section 3.4). $\mathrm{RCR}$ is delineated by the solid black line. It is shown that this region is more restricted than either the field of temperature inferred from the red and infrared local emission rate. The latter especially encompasses regions of higher temperature gradient surrounding the area of higher temperature. Capturing these gradients can also be a major step towards providing high quality data for the validation of formation and oxidation models that are highly temperature dependent [7]. 
Thus, the MAE technique is able to recover a relatively wide range of soot temperature at a fairly low level of uncertainties when using one-color fields of spectral emission rate. This statement also highlights the original contribution of the present study.

\section{Conclusion}

A jointly theoretical and numerical approach has been outlined allowing the local spectral absorption coefficient and the local spectral emission rate to be retrieved inside a steady axis-symmetric laminar coflow non-premixed ethylene flame. Operating within two spectral ranges where the aforementioned fields can be attributed to soot, the methodology then enables the concomitant retrievals of the soot temperature and volume fraction maps. The methodology offers a self-calibration way to retrieve the temperature field over a region where the ratio of the blackbody intensities can be detected within both spectral ranges. This calibration also depends directly on the measurements of both local spectral absorption coefficient fields. Therefore, no assumption on the soot refractive index is required in this technique.

Using fields provided by a numerical simulation, the performance of the methodology has been assessed. This process allows the identification of the confidence regions. The confidence region related to the ratio of the blackbody intensities can be used as a calibration database for each local spectral emission rate, which in turn allows the temperature distribution to be inferred. Due to less intensive data manipulations, the temperature map as inferred from a one-color field exhibits a wider spatial extension at a similar level of uncertainties. Experimental evidence of these findings has 
been presented in the present paper.

The experimental setup has been specifically implemented to enable the technique. While the operating parameters, such as the spectral ranges, can be further optimized to improve the performances of the technique, the setup could now provide with experimental fields at a higher frame rate. This would allow the soot temperature field to be tracked, therefore gaining insights into the flame flickering phenomenon. Thus, the intermittent quenching at the flame tip that leads to soot release into the atmosphere could be studied to get better understanding.

Following an assessment similar to the one presented here, the technique could also be extended to turbulent, therefore non-axis-symmetric flames. This would require an interaction between numerical simulations, that would provide with the numerical data required to characterize the fields along the optical pathways, and the experimental maps, that would in turn allows the numerical model to be refined.

Eventually, extending the technique to a third wavelength could now allow the soot particles mean diameter to be mapped.

\section{Acknowledgments}

The authors feel grateful to Prof. Sagaut for his contribution to the fund-

ing of the experimental hardware and Dr. P. Gerlinger's group for sharing the outputs produced by their numerical simulation. 
[1] M. Patricia Sierra-Vargas, L. M. Teran, Respirology 17 (2012) 10311038.

[2] R.A. Kerr, Science 339 (6118) (2013) 382.

[3] M. Frenklach, H. Wang, Proc. Combust. Inst. 23 (1991) 1559-1566.

[4] I.M. Kennedy, C. Yam, D.C. Rapp, R.J. Santoro, Combust. Flame 107 (1996) 368-382.

[5] M.D. Smooke, C.S. McEnally, L.D. Pfefferle, R.J. Hall., M.B. Colket, Combust. Flame 117 (1999) 117-139.

[6] S.J. Brookes, J.B. Moss, Combust. Flame 116 (1999) 486-503.

[7] G. Blanquart, H. Pitsch, Combust. Flame 156 (2009) 1614-1626.

[8] F. Liu, H. Guo, G.J. Smallwood, Ö.L. Gülder, Combust. Theory Modelling 7 (2003) 301-315.

[9] C.S. McEnally, L.D. Pfefferle, Combust. Flame 148 (2007) 210-222.

[10] M. Kashif, P. Guibert, J. Bonnety, G. Legros, Combust. Flame 161 (2014) 1575-1586.

[11] M. Kashif, J. Bonnety, A. Matynia, P. Da Costa, G. Legros, Combust. Flame (2015) in press, DOI: 10.1016/j.combustflame.2014.12.005.

[12] J.-L. Consalvi, F. Liu, J. Contreras, M. Kashif, G. Legros, S. Shuai, J. Wang, Combust. Flame 162 (2015) 1153-1163. 
[13] S. Schraml, S. Dankers, K. Bader, S. Will, A. Leipertz, Combust. Flame 120 (2000) 439-450.

[14] F. Liu, D.R. Snelling, K.A. Thomson, G.J. Smallwood, Appl Phys B 96 (2009) 623-636.

[15] F. Goulay, P.E. Schrader, H.A. Michelsen, Appl Phys B 100 (2010) 655-663.

[16] Y.R. Sivathanu, G.M. Faeth, Combust. Flame 81 (1990) 150-165.

[17] F. Xu, K.-C. Lin, G.M. Faeth, Combust. Flame 115 (1998) 195-209.

[18] P.B. Kuhn, B. Ma, B.C. Connelly, M.D. Smooke, M.B. Long, Proc. Combust. Inst. 33 (2011) 743-750.

[19] F. Liu, K.A. Thomson, G.J. Smallwood, Combust. Flame 160 (2013) 1693-1705.

[20] H. Zhao, B. Williams, R. Stone, J. Quant. Spectrosc. Radiat. Transf. 133 (2014) 136-152.

[21] C. Arcoumanis, C. Bae, A. Nagwaney, J.H. Whitelaw, SAE Paper (1995) 950-850.

[22] H.X. Quoc, J.-M. Vignon, M. Brun, SAE Paper (1991) 910-736.

[23] T.P. Jenkins, R.K. Hanson, Combust. Flame 126 (2001) 1669-1679.

[24] T. Blacha, M. Di Domenico, P. Gerlinger, M. Aigner, Combust. Flame 159 (2012) 181-193. 
[25] M.F. Modest, Radiative Heat Transfer (second edition) Academic Press, USA, 2003, p. 263.

[26] M. Kashif, J. Bonnety, P. Guibert, C. Morin, G. Legros, Opt. Exp. 20 (2012) 28742-28751.

[27] G. Legros, A. Fuentes, J. Baillargeat, P. Joulain, J.P. Vantelon, J.L. Torero, Opt. Lett. 30 (2005) 3311-3313.

[28] K. J. Daun, K. A. Thomson, F. Liu, G. J. Smallwood, Appl. Opt. 45 (2006) 4638-4646.

[29] E. Akesson, K.J. Daun, Appl. Opt. 47 (2008) 407-416.

[30] R. J. Hall, P.A. Bonczyk, Appl. Opt. 29 (1990) 4590-4598.

[31] F. Cignoli, S. De Iuliis, V. Manta, G. Zizak, Appl. Opt. 40 (2001) 53705378.

[32] C.S. Mcenally, A.M. Schaffer, M.B. Long, L.D. Pfefferle, M.D. Smooke, M.B. Colket, R.J. Hall, Symp. (Intl.) Combust. (1998) 1497-1505.

[33] T.F. Coleman, Y.Y. Li, SIAM J. Optimiz. 6 (1996) 418-445.

[34] H. Chang, T.T. Charalampopoulos, Proc. R. Soc. London 430 (1990) $577-591$.

[35] J.E. Farrell, P.B. Catrysse, B.A Wandell, Appl. Opt. 51 (2012) A80-A90.

[36] G. Legros, T. Gomez, M. Fessard, T. Gouache, T. Ader, P. Guibert, P. Sagaut, J.L. Torero, Proc. Combust. Inst. 33 (2011) 1095-1103. 
[37] C. McEnally, Ü.Ö. Koöylü, L. Pfefferle, D. Rosner, Combust. Flame 109 (1997) 701-720.

[38] R. Santoro, T. Yeh, J. Horvath, H. Semerjian, Combust. Sci. Technol. 53 (1987) 89-115.

[39] C.P. Arana, M. Pontoni, Combust. Flame 138 (2004) 362-372. 


\section{List of Figures}

1 Schematic of the optical pathway to a pixel on sensor $\mathrm{S}_{1}$. On the left, cross-section of an axis-symmetric flame at a given height above the burner. The fields of temperature $\mathrm{T}$ and spectral absorption coefficient $\kappa$ are schematically discretized. On the right, optical arrangement schematically composed of a set of convergent lenses $\mathrm{L}_{1}$ and $\mathrm{L}_{2}$ separated by a pinhole $\mathrm{P}_{1}$, allowing the flux $\phi$ to be captured on a row of pixels on $\mathrm{S}_{1} .49$

2 Schematic of half a flame cross section at a given height $z_{i}$ above the burner. The magnification ratio on sensor $\mathrm{S}_{1}$ is here set to unity. $S_{1}$ is represented by half a row of pixels starting from the bottom at the location of the flame's axis projected on $\mathrm{S}_{1} \cdot\left(I_{i j}\right)_{j=1, N}$ are the signals provided by the $N$ pixels in this half a row $(N=6$ here $) . \ldots \ldots \ldots$

3 Retrieved data at wavelength $\lambda_{1}$ (black dots) delivered by the MAE technique following Eqs.(10) and (16) as functions of the original data provided by the numerical simulation of Blacha et al. [24]: on the left, local spectral absorption coefficients; on the right, local spectral emission rates. The blue line indicates the ideal location of the retrieved data. No noise was included within the processing. . . . . . . . . . . . . 51 
4 Retrieved data (black dots) delivered by the MAE technique following Eqs.(10) and (16) as functions of the original data provided by the numerical simulation of Blacha et al. [24]: on the left, soot volume fraction inferred from the red absorption coefficient; on the right, temperature as inferred from the ratio of the blackbody intensities. The blue line indicates the ideal location of the retrieved data. Before processing, the fields of theoretical signals have been perturbated by a gaussian noise with a zero mean and a standard deviation of 50 . The absolute errors ( $\mathrm{AE}$ ) between the original and retrieved data have been averaged over the whole fields. . . . . . . . . . . . . . 52

5 Maps of soot volume fraction (upper row) and soot temperature (lower row). On the left, original data provided by the numerical simulation of Blacha et al. [24]. In the middle, retrieved data delivered by the MAE technique. The soot volume fraction and the soot temperature retrieved are inferred from the red absorption coefficient and the ratio of the blackbody intensities, respectively. On the right, contours defined by some isovalues of the absolute discrepancies between the original and retrieved data. . . . . . . . . . . . . . 53 
6 Evolution of the mean absolute discrepancy between the original and retrieved temperatures as a function of the original field that allow the temperature field to be retrieved. The original field is either the red local emission rate (red solid line) or the infrared local emission rate (black solid line), or the ratio of the blackbody intensities (blue solid line). Every axis has been normalized by the peak value met in the corresponding field. The red and black circles indicate the thresholds of the red and infrared local emission rates, respectively, above which the mean discrepancy is lower than $40 \mathrm{~K}$. . . . . . . . . . . 54

7 Distribution of the mean absolute discrepancy between the original and retrieved temperatures. Here, the latter have been inferred from the ratio of the blackbody intensities. The size of any population has been normalized by the number of data considered. These stand either in the ratio confidence region (red triangles) or in the whole field (blue circles) provided that the data exhibit values within the ranges specified by the

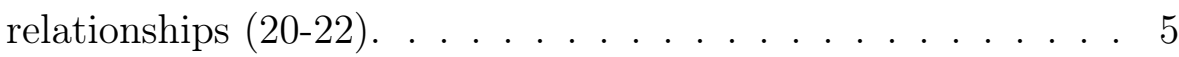

8 Maps of soot temperature as inferred from the retrieved local (a) red and (b) infrared emission rates. Only the temperatures exhibiting an absolute discrepancy with the original temperatures lower than $\pm 40 \mathrm{~K}$ are mapped. The dotted contour delineates the ratio confidence region that encompasses the calibrating data. . . . . . . . . . . . . . 56 
9 Schematic of the optical arrangement for the two-dimensional modulated absorption/emission technique. The inserts show typical frames captured by the cameras that image the red (on the left) and infrared (on the right) signals when the shutters are open and the flame is established. The shadow of the fuel duct can be seen at the bottom of each frame. . . . . . . . . 57

10 Evolution along the visible spectrum of the power delivered by the calibrating lamp. The shaded bands indicate the transmissivity width of the red and infrared filters mounted on the cameras. . . . . . . . . . . . . . . . . 58

11 Evolution with temperature of the ratio of the blackbody intensities as captured over the red and infrared spectral ranges. The ratio is corrected for the discrepancy $\chi$ of the light collection along the red and infrared pathways. The solid line represents the evolution for the mean $\chi=1.359$ measured. The dotted lines are the evolutions followed when $\chi$ is altered by

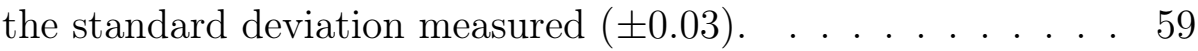

12 Maps of computed (left column) and experimental (right column) signals, for both red (upper row) and infrared (lower row) spectral ranges. For each map, the location of the maximum intensity $I_{\max }$ is $\left(r_{\max }, z_{\max }\right) \ldots \ldots \ldots$. . . . . . . 60 
13 Profiles of soot volume fraction at different heights above the burner. The shaded areas represent the region where the uncertainties are higher than the error bar indicated in the upper right corner. Blue solid line: numerical data from Blacha et al. [24]; red stars $(*)$ : experimental data from Arana et al. [39]; black downward triangles $(\nabla)$ : experimental data provided by the MAE technique in the red spectral range; green upward triangles $(\triangle)$ : experimental data provided by the MAE technique in the infrared spectral range. . . . . . . . . . . . . . . 61

14 Maps of soot local spectral absorption coefficient: (a) measured red coefficients; (b) measured infrared coefficients; (c) infrared coefficients as modeled following the expression by Chang and Charalampopoulos [34] that has been calibrated with the peak red absorption coefficient measured. The contours are identified by the values indicated in the color bar. . . 62 
15 Profiles of soot temperature at different heights above the burner. Blue solid line: numerical data from Blacha et al. [24]; red stars $(*)$ : experimental data from McEnally et al. [32]; purple circles (o): experimental temperature inferred from the ratio of the blackbody intensities; black downward triangles $(\nabla)$ : experimental temperature inferred from the red emission rate; green upward triangles $(\triangle)$ : experimental temperature inferred from the infrared emission rate. The shaded areas represent the regions located outside the ratio confidence region. In these shaded areas, the temperature inferred from the ratio of the blackbody intensities exhibits an uncertainty level higher than the error bar indicated in the upper right corner. . . . . . . . . . . . . . . . . 63

16 Maps of soot temperature as inferred from the retrieved local (a) red and (b) infrared emission rates. Only the measurements inferred from spectral emission rates higher than the thresholds indicated in Fig.6 are shown. The ratio confidence region encompassing the calibrating data is delineated by the black solid line. . . . . . . . . . . . . . . . . . . . . . . 64 


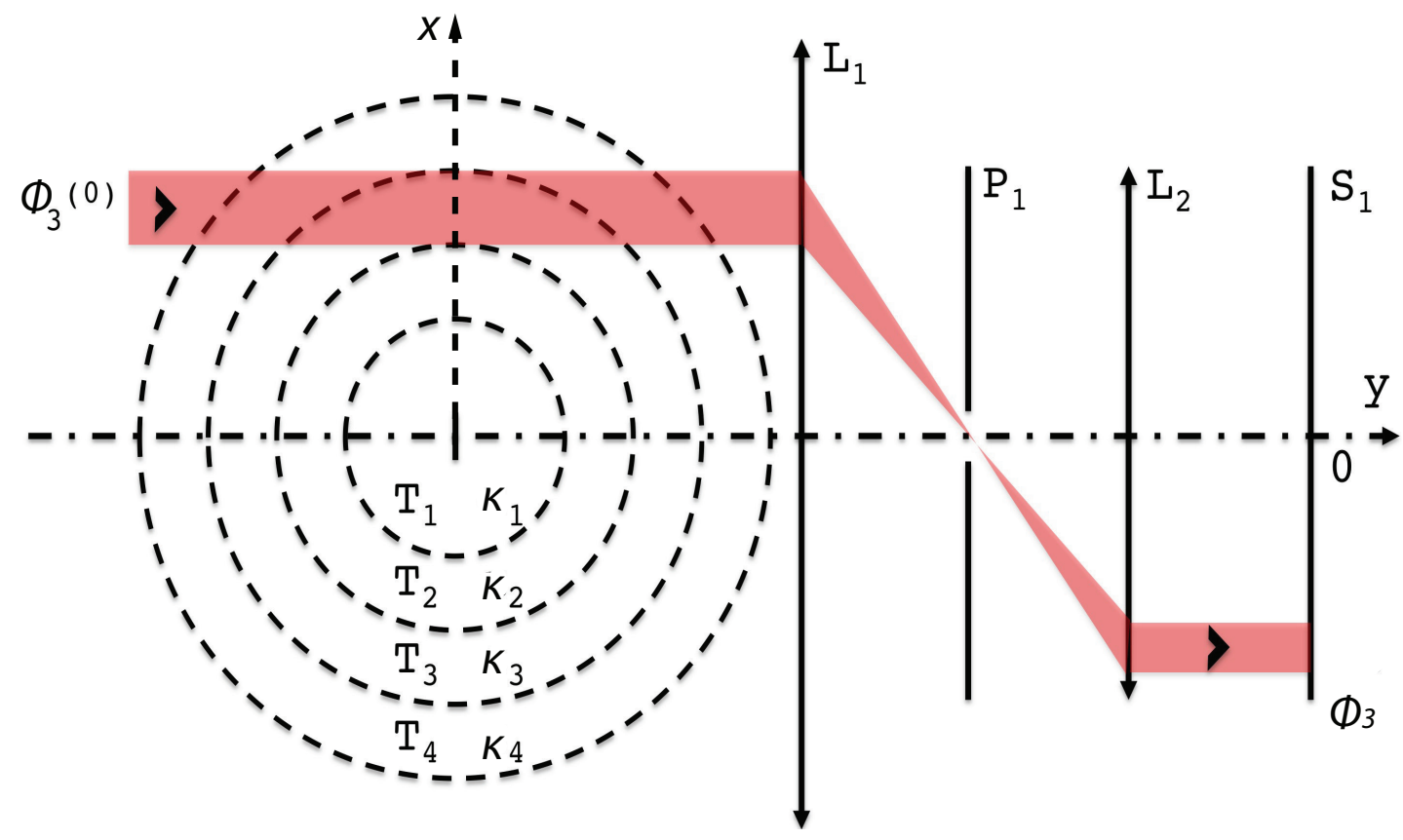

Figure 1: Schematic of the optical pathway to a pixel on sensor $\mathrm{S}_{1}$. On the left, crosssection of an axis-symmetric flame at a given height above the burner. The fields of temperature $\mathrm{T}$ and spectral absorption coefficient $\kappa$ are schematically discretized. On the right, optical arrangement schematically composed of a set of convergent lenses $\mathrm{L}_{1}$ and $\mathrm{L}_{2}$ separated by a pinhole $\mathrm{P}_{1}$, allowing the flux $\phi$ to be captured on a row of pixels on $\mathrm{S}_{1}$. 


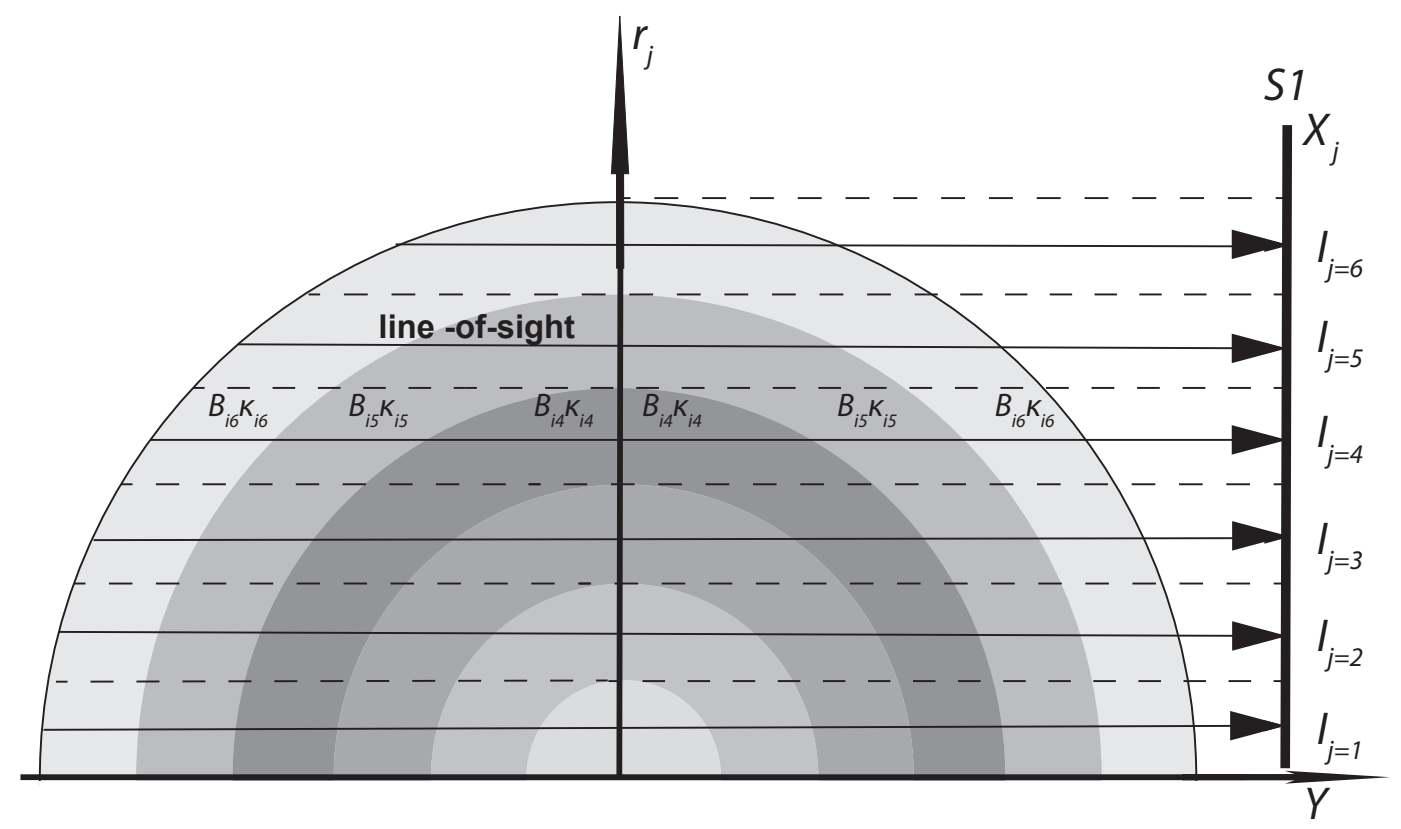

Figure 2: Schematic of half a flame cross section at a given height $z_{i}$ above the burner. The magnification ratio on sensor $S_{1}$ is here set to unity. $S_{1}$ is represented by half a row of pixels starting from the bottom at the location of the flame's axis projected on $\mathrm{S}_{1}$. $\left(I_{i j}\right)_{j=1, N}$ are the signals provided by the $N$ pixels in this half a row $(N=6$ here). 


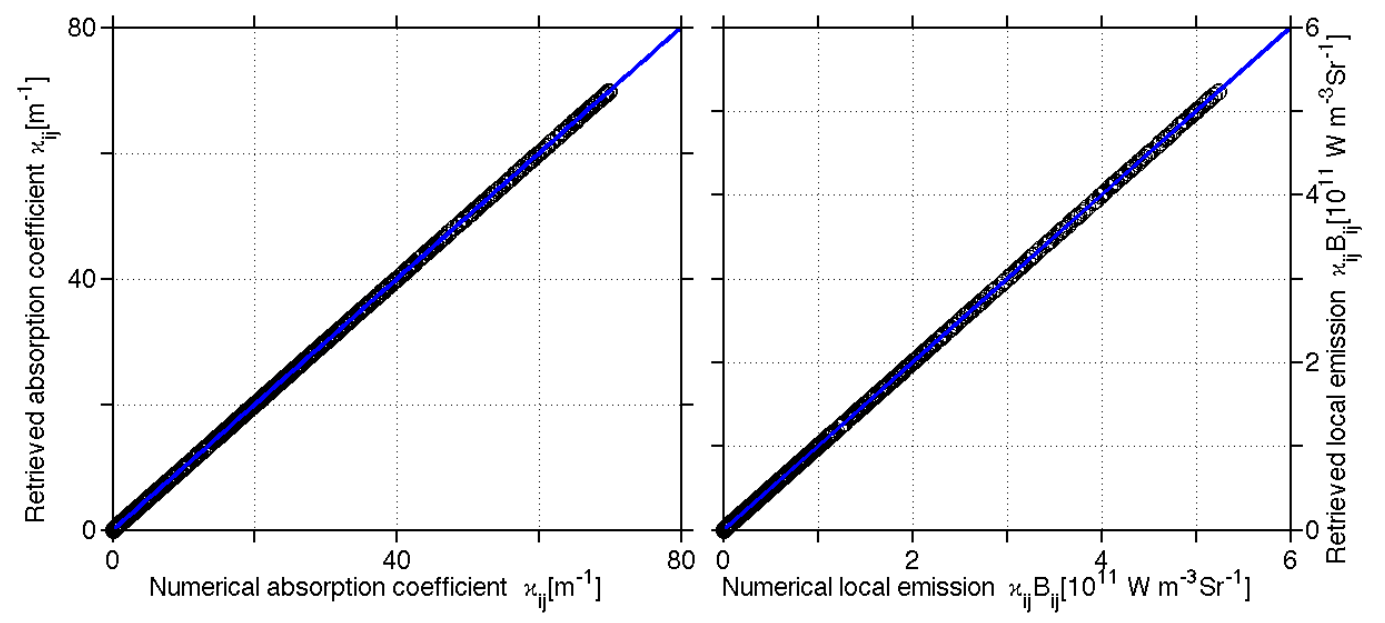

Figure 3: Retrieved data at wavelength $\lambda_{1}$ (black dots) delivered by the MAE technique following Eqs.(10) and (16) as functions of the original data provided by the numerical simulation of Blacha et al. [24]: on the left, local spectral absorption coefficients; on the right, local spectral emission rates. The blue line indicates the ideal location of the retrieved data. No noise was included within the processing. 


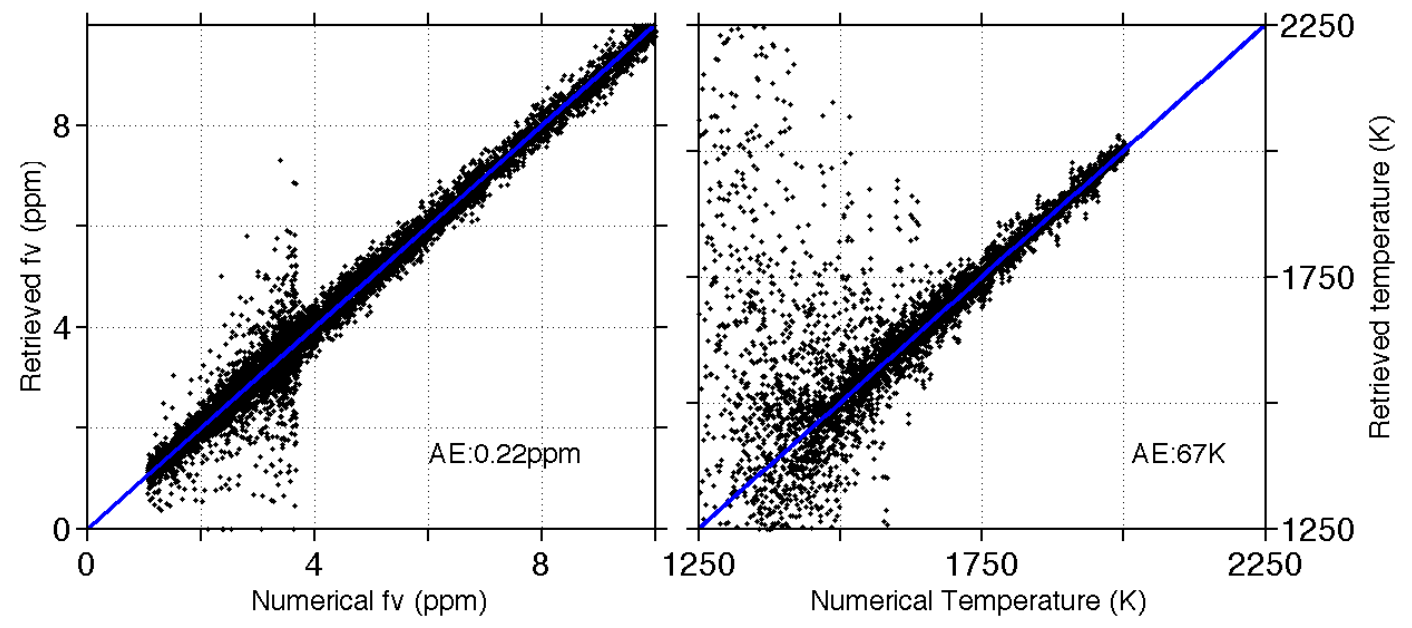

Figure 4: Retrieved data (black dots) delivered by the MAE technique following Eqs.(10) and (16) as functions of the original data provided by the numerical simulation of Blacha et al. [24]: on the left, soot volume fraction inferred from the red absorption coefficient; on the right, temperature as inferred from the ratio of the blackbody intensities. The blue line indicates the ideal location of the retrieved data. Before processing, the fields of theoretical signals have been perturbated by a gaussian noise with a zero mean and a standard deviation of 50 . The absolute errors (AE) between the original and retrieved data have been averaged over the whole fields. 

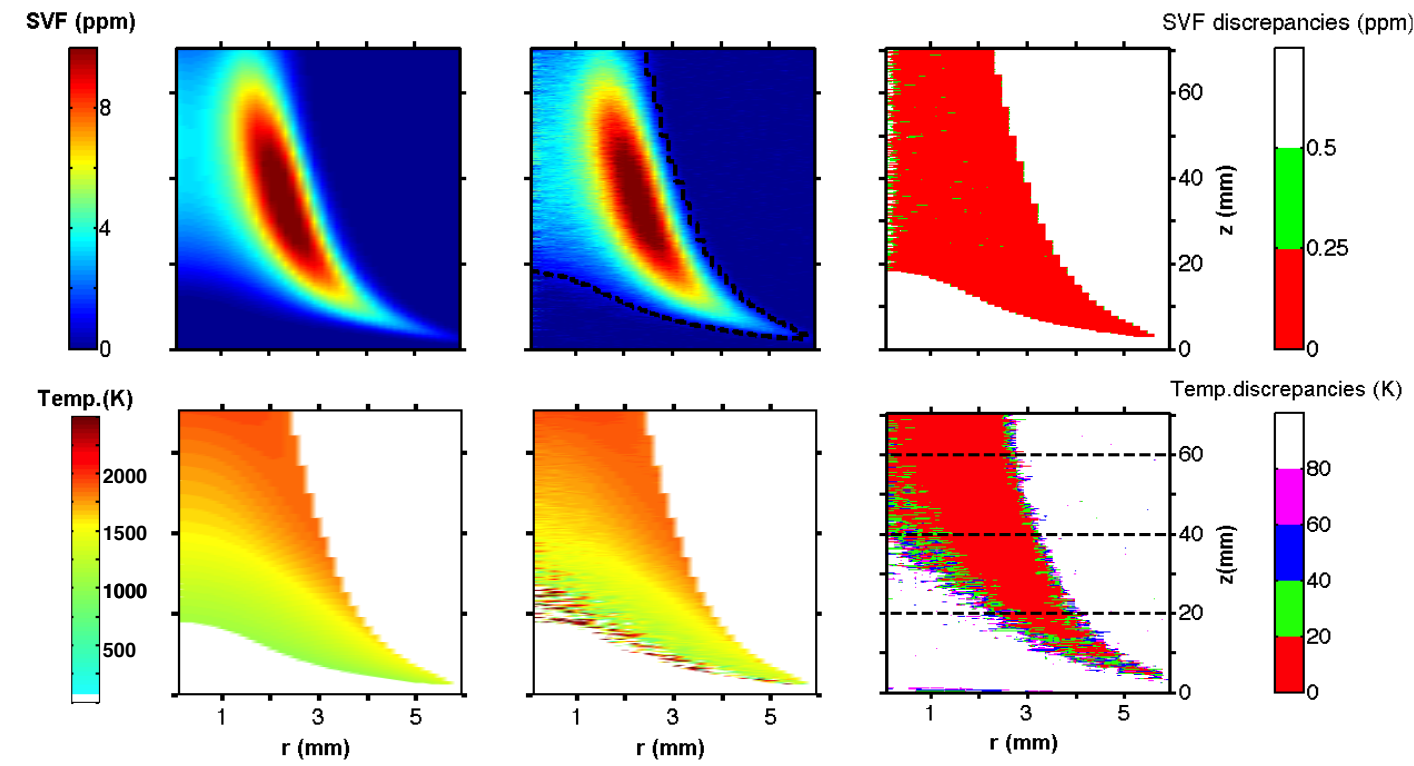

Figure 5: Maps of soot volume fraction (upper row) and soot temperature (lower row). On the left, original data provided by the numerical simulation of Blacha et al. [24]. In the middle, retrieved data delivered by the MAE technique. The soot volume fraction and the soot temperature retrieved are inferred from the red absorption coefficient and the ratio of the blackbody intensities, respectively. On the right, contours defined by some isovalues of the absolute discrepancies between the original and retrieved data. 


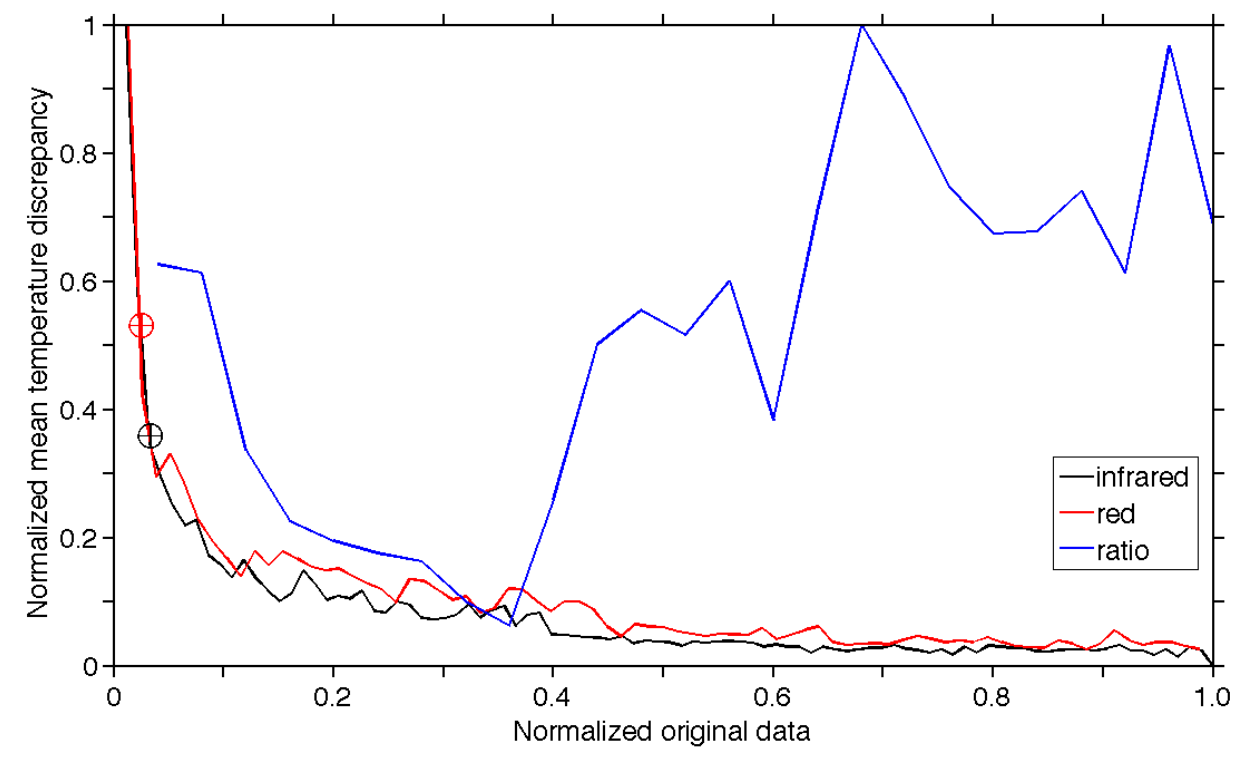

Figure 6: Evolution of the mean absolute discrepancy between the original and retrieved temperatures as a function of the original field that allow the temperature field to be retrieved. The original field is either the red local emission rate (red solid line) or the infrared local emission rate (black solid line), or the ratio of the blackbody intensities (blue solid line). Every axis has been normalized by the peak value met in the corresponding field. The red and black circles indicate the thresholds of the red and infrared local emission rates, respectively, above which the mean discrepancy is lower than $40 \mathrm{~K}$. 


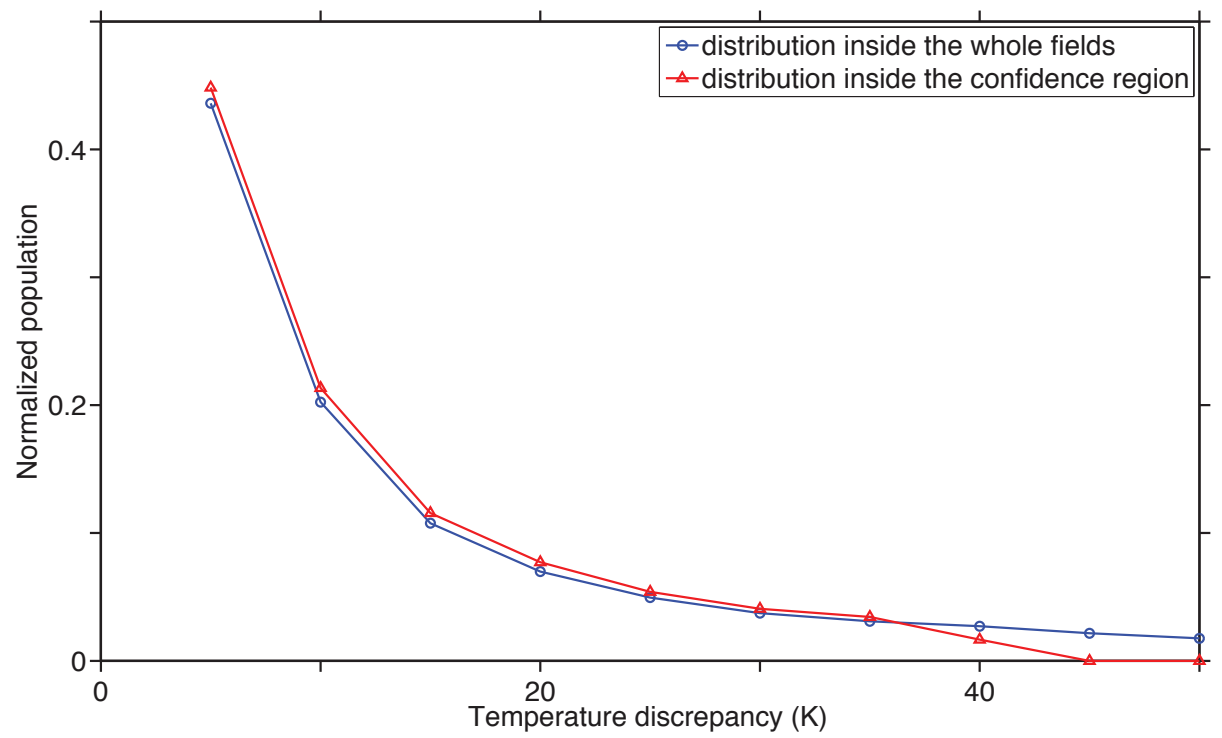

Figure 7: Distribution of the mean absolute discrepancy between the original and retrieved temperatures. Here, the latter have been inferred from the ratio of the blackbody intensities. The size of any population has been normalized by the number of data considered. These stand either in the ratio confidence region (red triangles) or in the whole field (blue circles) provided that the data exhibit values within the ranges specified by the relationships (20-22). 

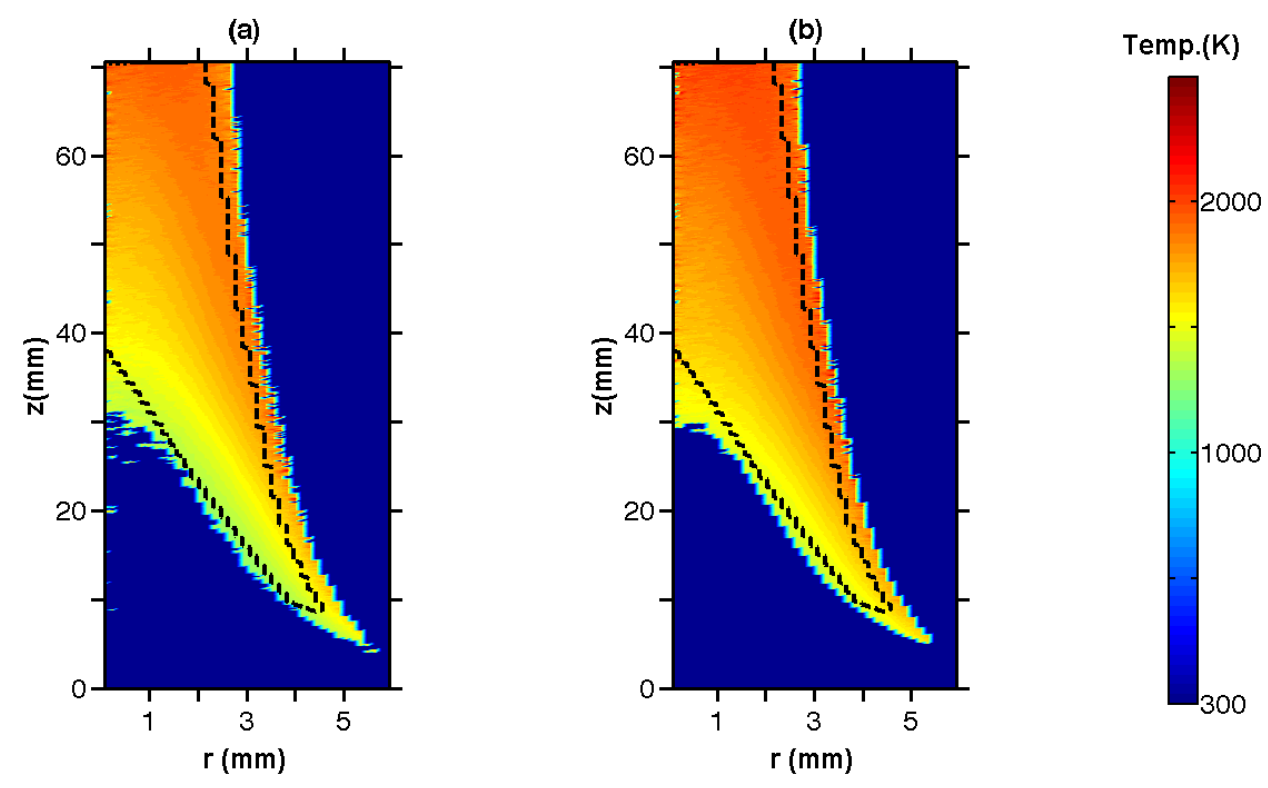

Figure 8: Maps of soot temperature as inferred from the retrieved local (a) red and (b) infrared emission rates. Only the temperatures exhibiting an absolute discrepancy with the original temperatures lower than $\pm 40 \mathrm{~K}$ are mapped. The dotted contour delineates the ratio confidence region that encompasses the calibrating data. 


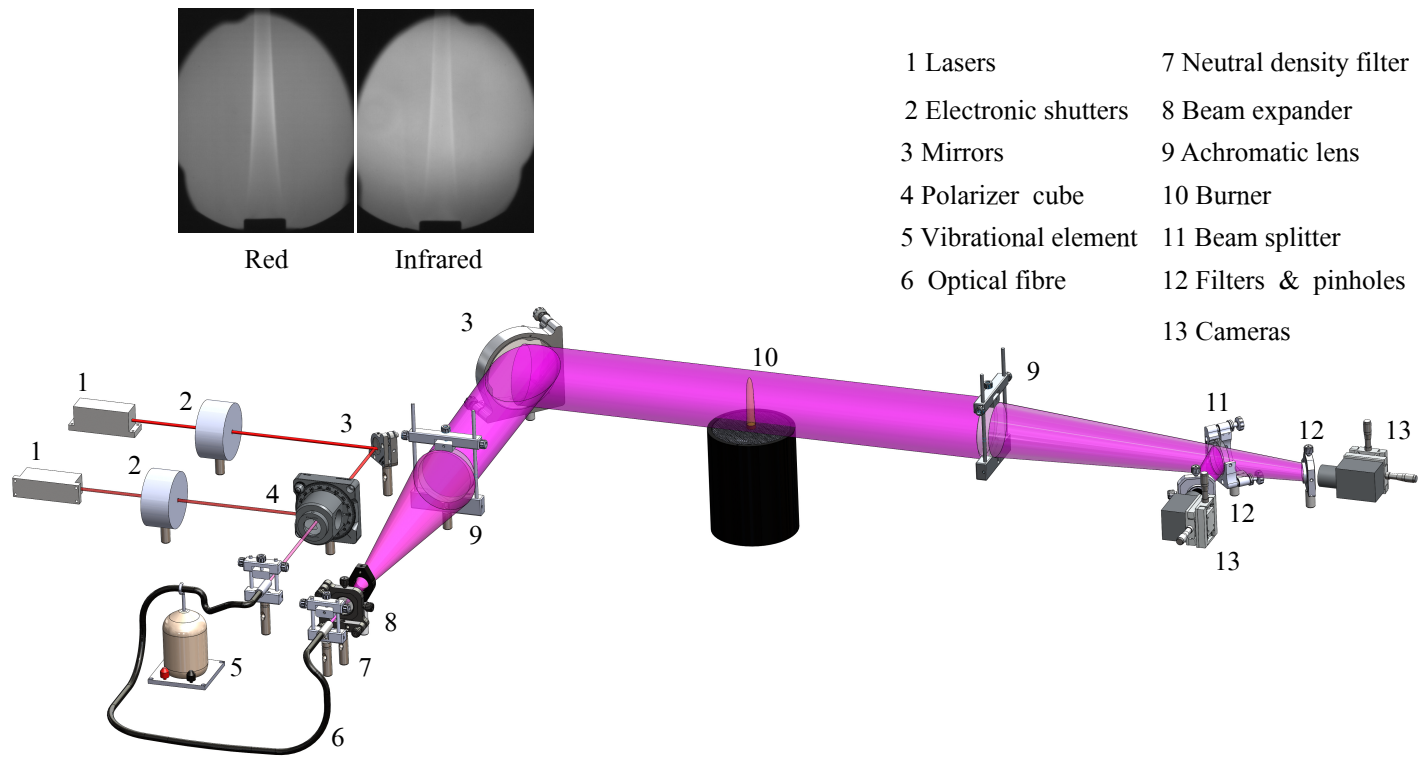

Figure 9: Schematic of the optical arrangement for the two-dimensional modulated absorption/emission technique. The inserts show typical frames captured by the cameras that image the red (on the left) and infrared (on the right) signals when the shutters are open and the flame is established. The shadow of the fuel duct can be seen at the bottom of each frame. 


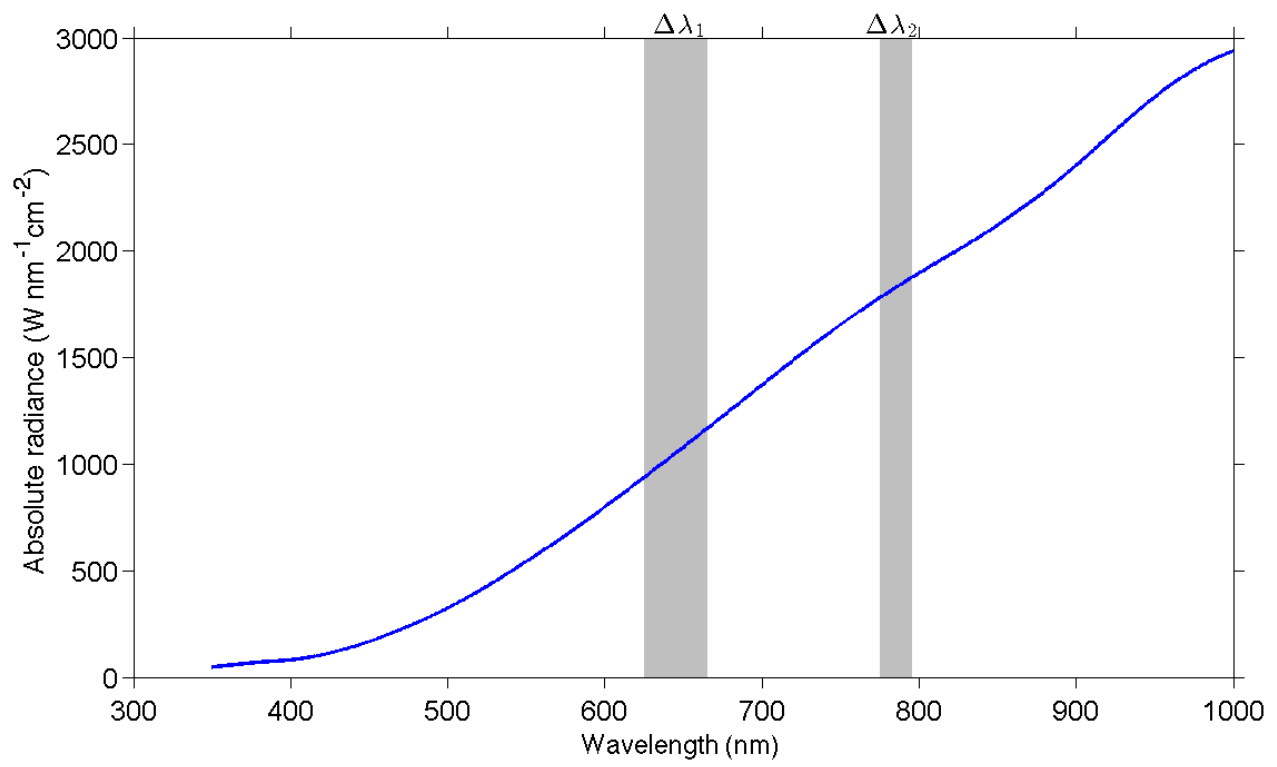

Figure 10: Evolution along the visible spectrum of the power delivered by the calibrating lamp. The shaded bands indicate the transmissivity width of the red and infrared filters mounted on the cameras. 


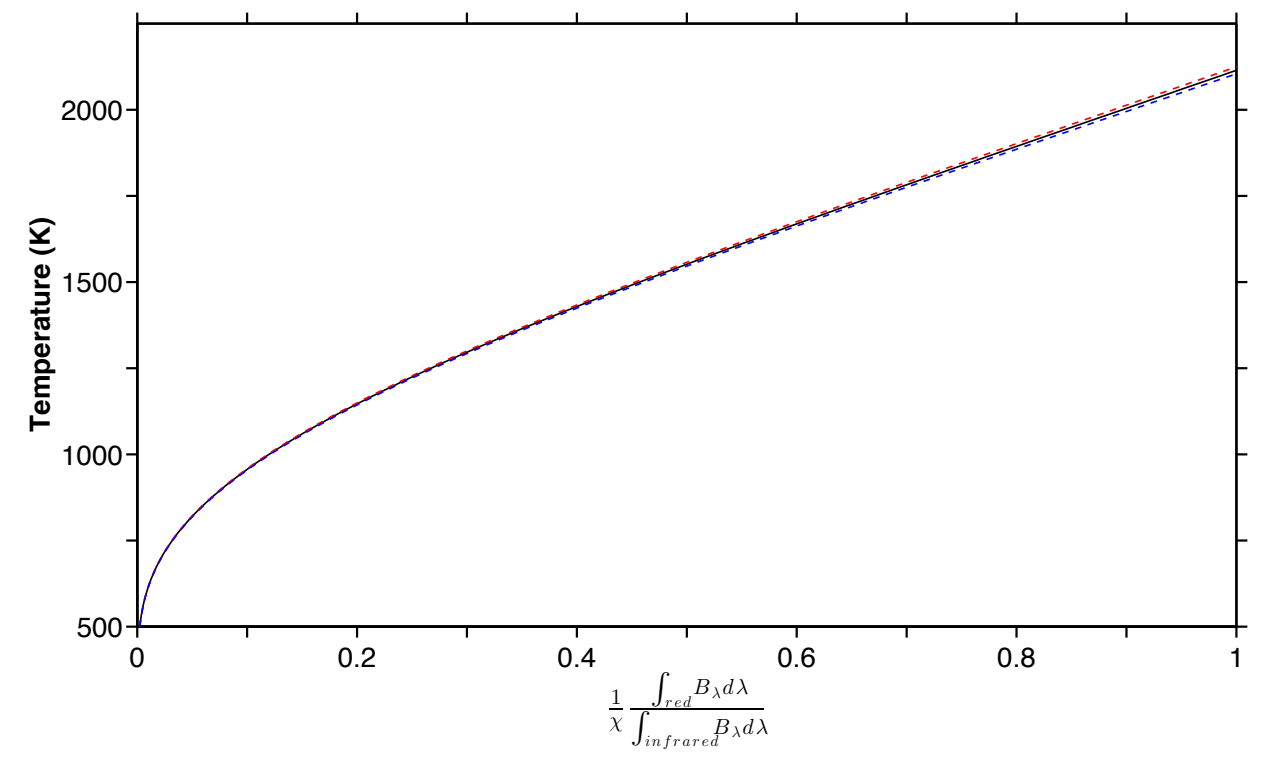

Figure 11: Evolution with temperature of the ratio of the blackbody intensities as captured over the red and infrared spectral ranges. The ratio is corrected for the discrepancy $\chi$ of the light collection along the red and infrared pathways. The solid line represents the evolution for the mean $\chi=1.359$ measured. The dotted lines are the evolutions followed when $\chi$ is altered by the standard deviation measured $( \pm 0.03)$. 


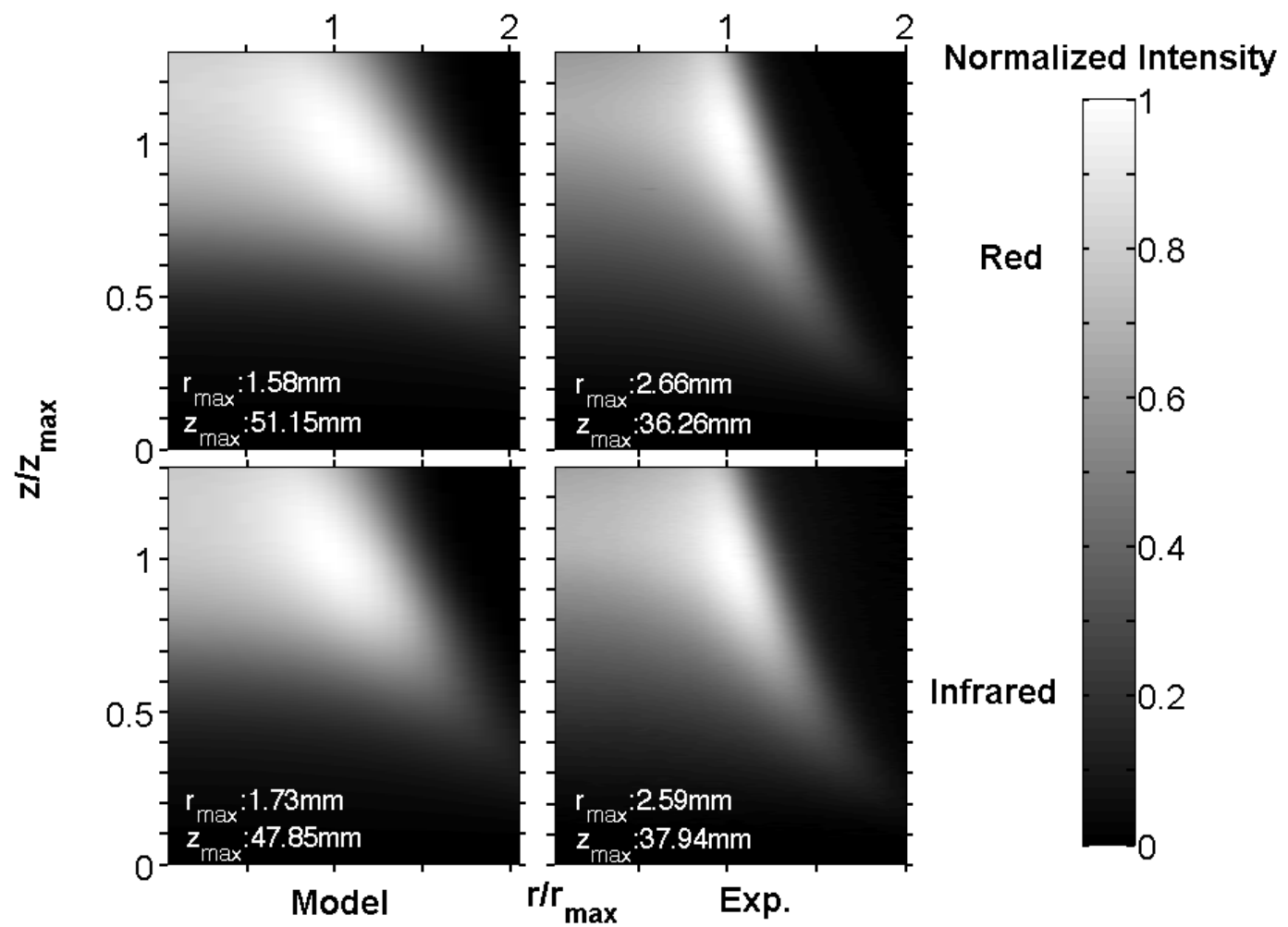

Figure 12: Maps of computed (left column) and experimental (right column) signals, for both red (upper row) and infrared (lower row) spectral ranges. For each map, the location of the maximum intensity $I_{\max }$ is $\left(r_{\max }, z_{\max }\right)$. 


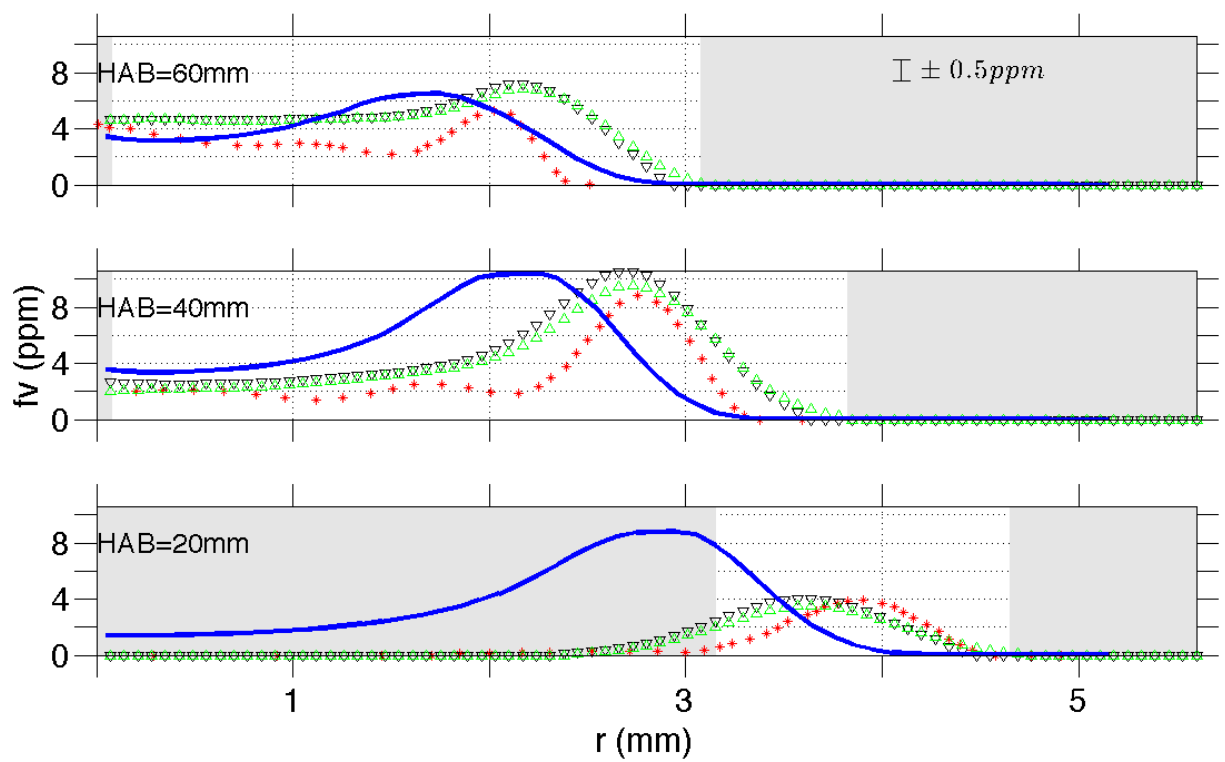

Figure 13: Profiles of soot volume fraction at different heights above the burner. The shaded areas represent the region where the uncertainties are higher than the error bar indicated in the upper right corner. Blue solid line: numerical data from Blacha et al. [24]; red stars $(*)$ : experimental data from Arana et al. [39]; black downward triangles $(\nabla)$ : experimental data provided by the MAE technique in the red spectral range; green upward triangles $(\triangle)$ : experimental data provided by the MAE technique in the infrared spectral range. 


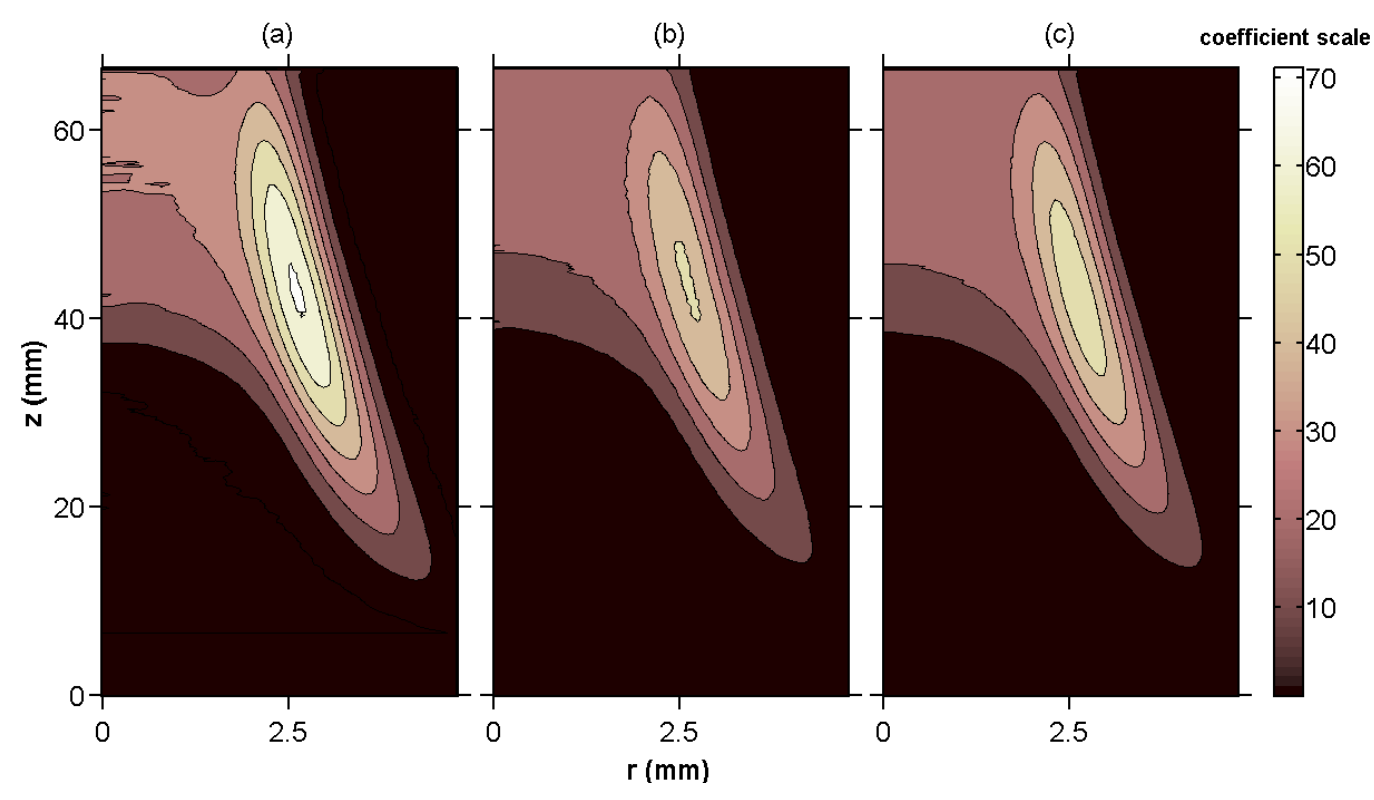

Figure 14: Maps of soot local spectral absorption coefficient: (a) measured red coefficients; (b) measured infrared coefficients; (c) infrared coefficients as modeled following the expression by Chang and Charalampopoulos [34] that has been calibrated with the peak red absorption coefficient measured. The contours are identified by the values indicated in the color bar. 


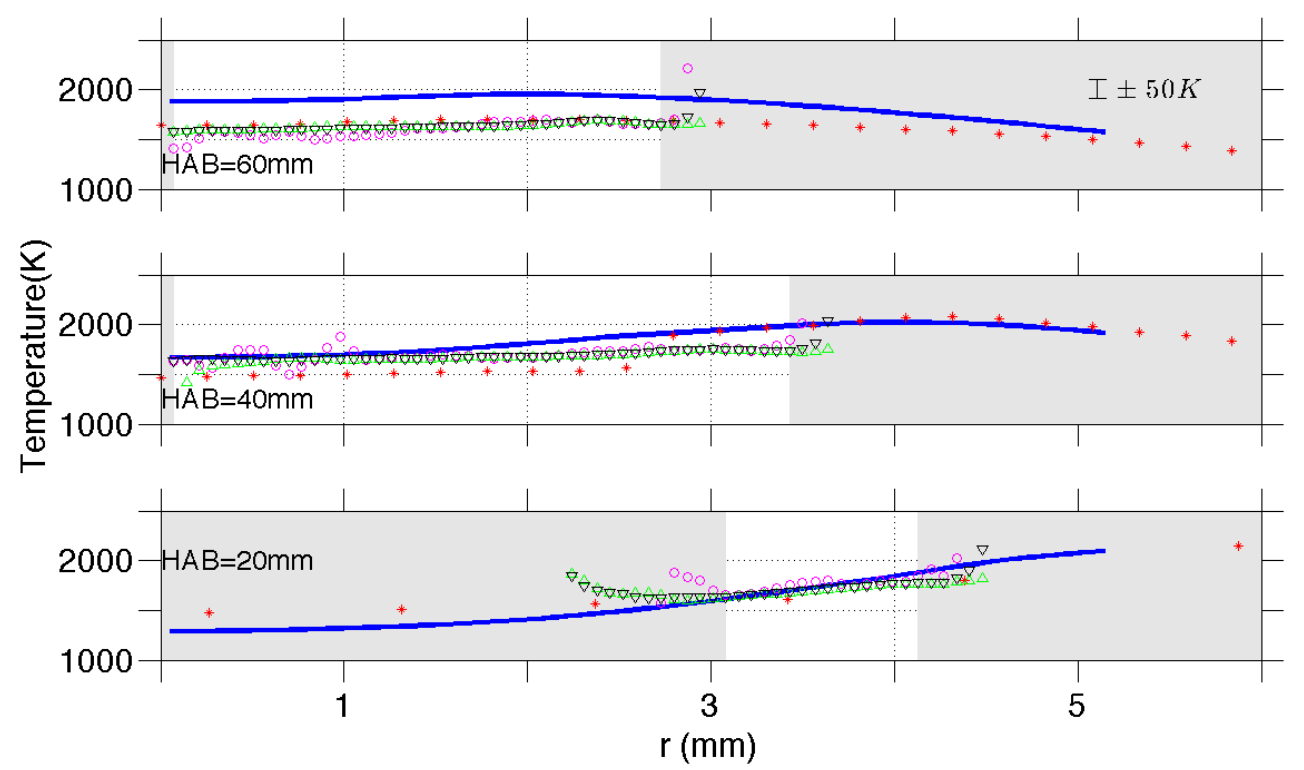

Figure 15: Profiles of soot temperature at different heights above the burner. Blue solid line: numerical data from Blacha et al. [24]; red stars $(*)$ : experimental data from McEnally et al. [32]; purple circles (o): experimental temperature inferred from the ratio of the blackbody intensities; black downward triangles $(\nabla)$ : experimental temperature inferred from the red emission rate; green upward triangles $(\triangle)$ : experimental temperature inferred from the infrared emission rate. The shaded areas represent the regions located outside the ratio confidence region. In these shaded areas, the temperature inferred from the ratio of the blackbody intensities exhibits an uncertainty level higher than the error bar indicated in the upper right corner. 

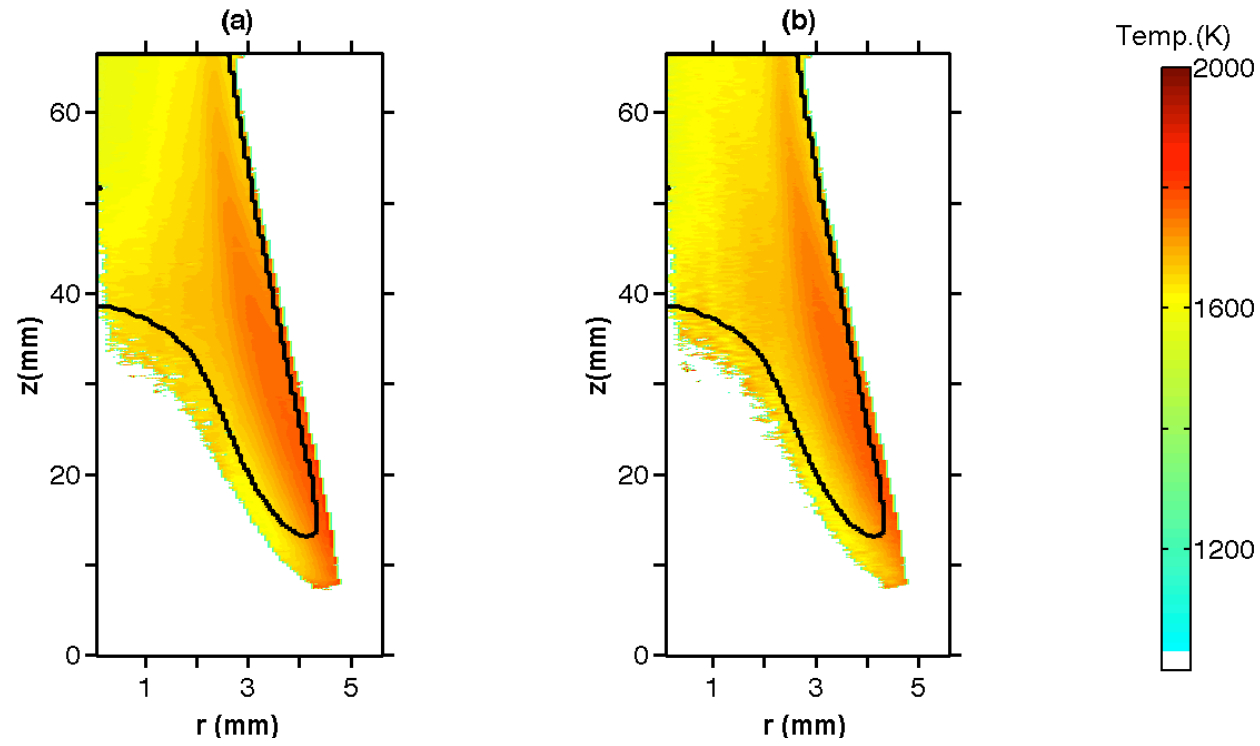

Figure 16: Maps of soot temperature as inferred from the retrieved local (a) red and (b) infrared emission rates. Only the measurements inferred from spectral emission rates higher than the thresholds indicated in Fig.6 are shown. The ratio confidence region encompassing the calibrating data is delineated by the black solid line. 